\title{
Dr.Paso:
}

\section{Drug response prediction and analysis system for oncology research}

Francisco Azuaje ${ }^{1,+,{ }^{*},}$, Tony Kaoma², Céline Jeanty, Petr V. Nazarov, Arnaud Muller, Sang-Yoon Kim, Anna Golebiewska ${ }^{1}$, Gunnar Dittmar², Simone P. Niclou¹.

${ }^{1}$ NorLux Neuro-Oncology Laboratory, Department of Oncology, Luxembourg Institute of Health (LIH), Luxembourg, Luxembourg.

${ }^{2}$ Proteome and Genome Research Unit, Department of Oncology, Luxembourg Institute of Health (LIH), Luxembourg, Luxembourg.

+ Current affiliation: ${ }^{2}$

*Corresponding Author, email: Francisco.Azuaje@lih.lu 


\section{Summary}

The prediction of anticancer drug response is crucial for achieving a more effective and precise treatment of patients. Models based on the analysis of large cell line collections have shown potential for investigating drug efficacy in a clinically-meaningful, cost-effective manner. Using data from thousands of cancer cell lines and drug response experiments, we propose a drug sensitivity prediction system based on a 47-gene expression profile, which was derived from an unbiased transcriptomic network analysis approach. The profile reflects the molecular activity of a diverse range of cancer-relevant processes and pathways. We validated our model using independent datasets and comparisons with published models. A high concordance between predicted and observed drug sensitivities was obtained, including additional validated predictions for four glioblastoma cell lines and four drugs. Our approach can accurately predict anti-cancer drug sensitivity and will enable further preclinical research. In the longer-term, it may benefit patient-oriented investigations and interventions. 


\section{Introduction}

The unbiased, large-scale prediction of anticancer drug activity using tumor-derived molecular data is crucial to deliver on the promise of a more personalized, precise treatment of cancer patients (Caponigro and Sellers, 2011; Ross and Wilson, 2014). The prediction of drug sensitivity based on the analysis of large collections of cell lines offers significant opportunities for investigating clinical efficacy in a biologically-meaningful, cost-effective manner (Geeleher et al., 2014; Goodspeed et al., 2016; Wilding and Bodmer, 2014). Computational models for predicting anticancer drug sensitivity can aid in the selection and prioritization of candidate compounds for pre-clinical research (Costello et al., 2014; Rees et al., 2016; Reinhold et al., 2012; Stetson et al., 2014).

Although cell line-based models may not fully recapitulate tumor biology, appropriately validated models may accelerate patient-oriented research, and have already shown potential to generate clinically-relevant predictions in different oncology domains. Such models may complement and in some cases offer an early substitute for in vivo models that tend to be expensive, time consuming and less scalable. In the short-term, this could enable the generation of novel biological hypotheses in the lab and, in the longer term, guide therapeutic decision-making in the clinic.

Over the past few years, the investigation of cell line-based computational models for anticancer drug sensitivity prediction has been accelerated by publicly-funded efforts of large research consortia (Barretina et al., 2012; lorio et al., 2016b; Reinhold et al., 2012; Yang et al., 2013). In particular, the Cancer Cell Line Encyclopedia (CCLE) (Barretina et al., 2012) and the Genomics of Drug Sensitivity in Cancer (GDSC) (Garnett et al., 2012; Yang et al., 
2013) projects represented significant steps forward for the oncology and pharmacogenomics research communities. These projects have generated and shared (untreated) molecular data from thousands of cancer cell lines and their accompanying treatment sensitivity measurements for hundreds of experimental and clinically-approved drugs. To date, computational models have mainly emphasized the application of different widely-investigated multivariable statistical and machine learning models, such as linear models and support vector machines, with various versions of feature selection methodologies (Dong et al., 2015; Haverty et al., 2016; Jang et al., 2014). Despite their potential for accurately predicting drug sensitivity across multiple types of cancer cell lines, less attention has been given to the investigation of biological importance of the proposed drug sensitivity markers, which have ranged from one to hundreds of gene-based features. Moreover, the majority of reported models have not been evaluated on independently generated datasets (Azuaje, 2017). Although different studies have tested the resulting prediction models on independent cell line datasets, e.g., models trained and tested on the GDSC and CCLE dataset respectively, there is a lack of studies that experimentally validate predicted anticancer sensitivity on independent biological samples, including cell lines that were not included in the training and initial testing datasets (Cortes-Ciriano et al., 2016; Gupta et al., 2016; Jang et al., 2014).

Here, we present Dr.Paso: Drug response prediction and analysis system for oncology research (Figure 1A). Dr.Paso predicts drug sensitivity responses based on the (baseline) expression patterns of 47 genes, which represented "hubs" in a pan-cancer transcriptomic network extracted from more than $1 \mathrm{~K}$ cell lines and are substantially implicated in a diversity of cancer-relevant biological processes. A computational prediction model based on the multiple-linear regression of the 47-gene expression values measured in hundreds of cell 
lines provided both accurate and robust prediction performance. First, the model was trained and cross-validated on a (discovery) dataset consisting of more than $10 \mathrm{~K}$ cell line-drug experiments for 24 (targeted and cytotoxic) drugs. Next, the resulting model was tested on a second, more recently-released, (validation) dataset comprising almost $10 \mathrm{~K}$ cell line-drug experiments that included 16 drugs also found in the discovery dataset. Dr.Paso's prediction performance is comparable to, and in some cases outperforms, previously published computational models. Motivated by these findings, Dr.Paso next predicted sensitivity scores for 4 glioblastoma (GBM) cell lines, including three (stem-like) cell lines that were not included in the discovery and validation datasets, against 24 drugs. We selected the top three drugs predicted as highly effective together with a drug predicted as lowly effective (negative control), and performed in vitro tests on the 4 cell lines. As in the case of the public datasets, the sensitivity scores estimated by Dr.Paso were highly concordant with the observed in vitro responses. To further facilitate research, we offer Dr.Paso through a Webbased interface that allows users to predict drug sensitivity scores for their own samples and expression data. The following sections will describe in detail these research phases, which are outlined in Figure 1B. 
A

Biological samples,

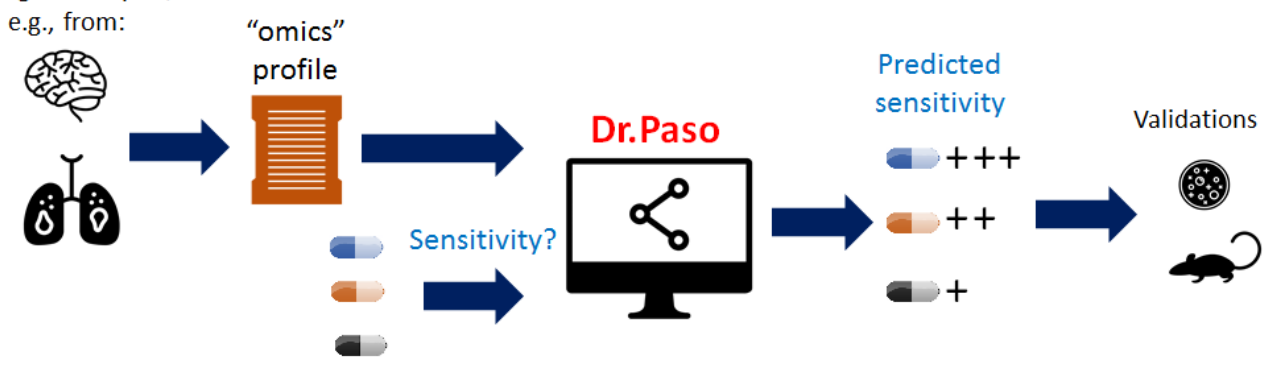

B

Discovery phase and prediction model generation

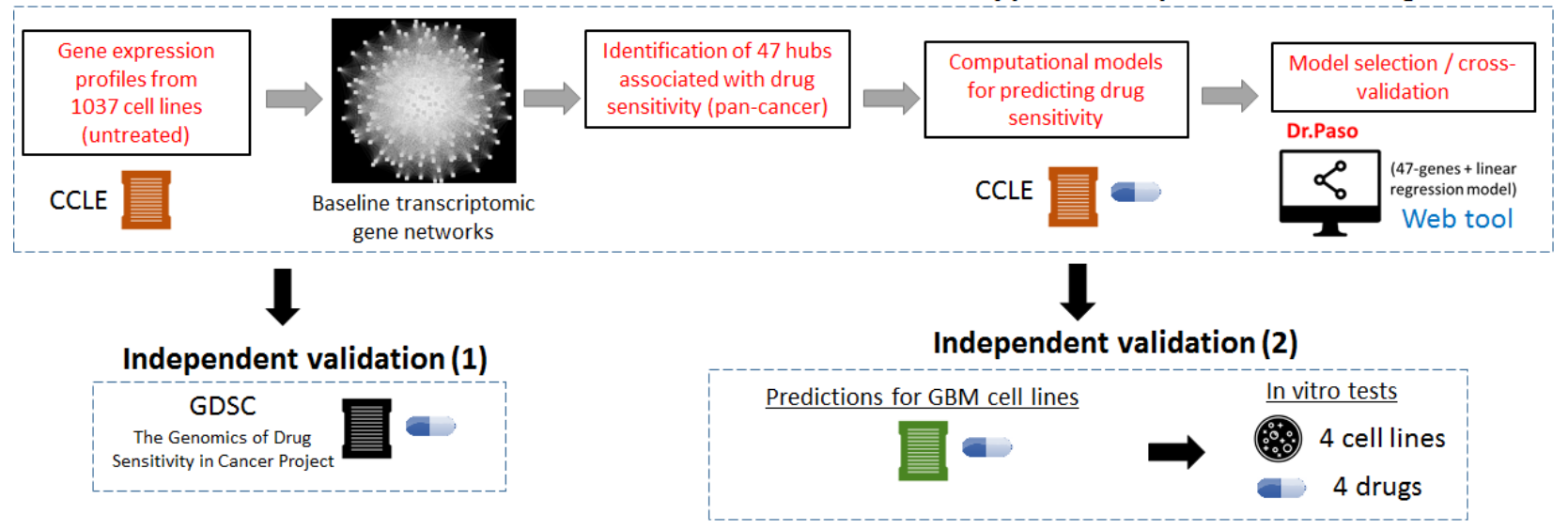

Figure 1. Dr.Paso: Overview of problem, application scenario and methodologies investigated. A. Outline of the general problem and application scenarios envisioned for the application of Dr.Paso. B. Workflow of the discovery phase, model generation and validation steps reported in this article.

\section{Results}

Hubs in a pan-cancer transcriptomic network display drug sensitivity predictive potential

Motivated by evidence indicating the drug sensitivity prediction power of gene expression profiles (Barretina et al., 2012; lorio et al., 2016a), we investigated the predictive potential 
of such data in the context of a pan-cancer transcriptomic correlation network. Our hypothesis was that genes highly connected within such networks, i.e., hubs, may be reflective of molecular activity across biological processes and tissue sites. To test this hypothesis, we analyzed the CCLE gene expression dataset, which was derived from 1037 (untreated) cell lines representing different cancer types in 18 tissue sites. To reduce network complexity while aiming at preserving potentially relevant information across all samples, we selected genes with highly variable expression pattern across cell lines (i.e., 177 genes with standard deviation of expression values across cell lines located above the 99 ${ }^{\text {th }}$ percentile). Using the pan-cancer expression profiles from these genes, we calculated all the between-gene (Pearson) correlation values and merged them into a fully-connected weighted network (Figure 2A), which included 177 nodes and more than $15 \mathrm{~K}$ edges (correlations).
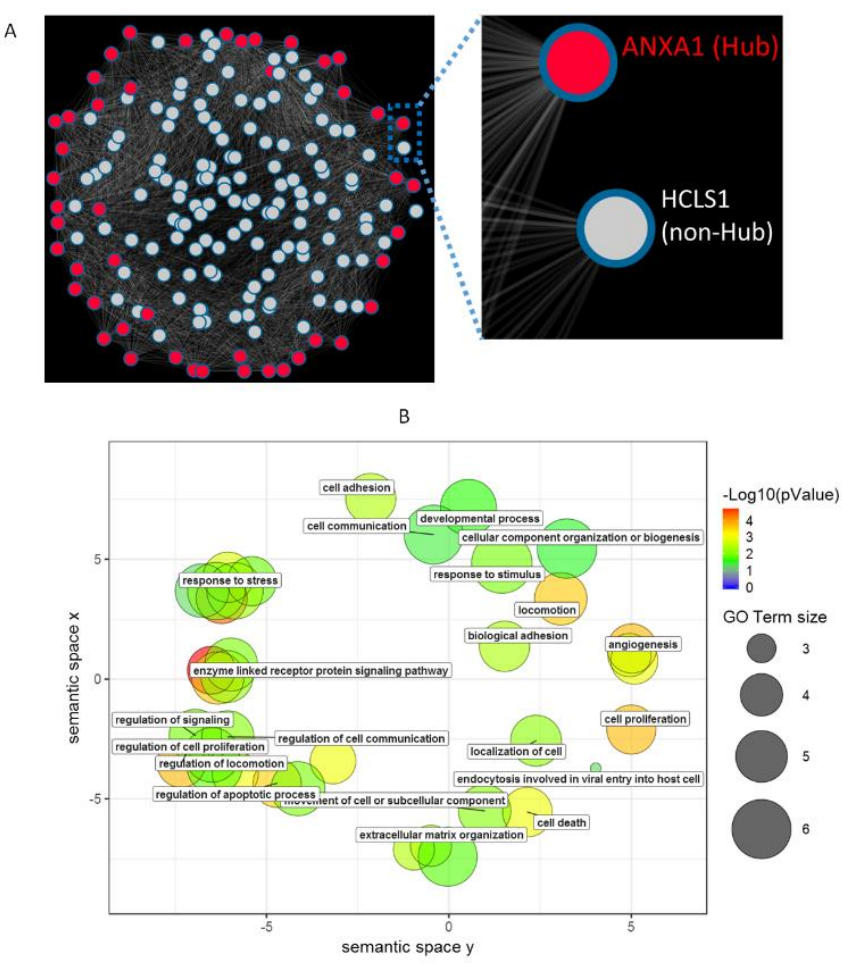

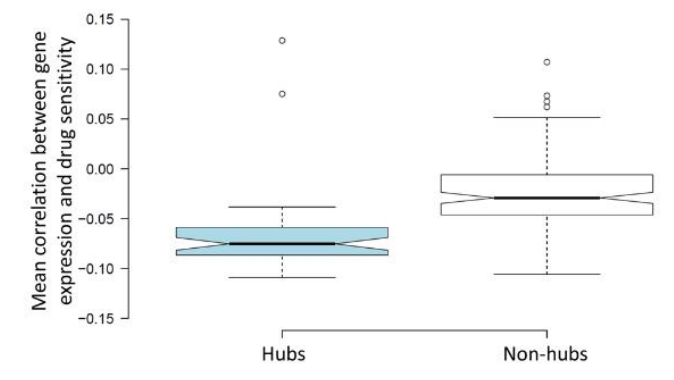

D

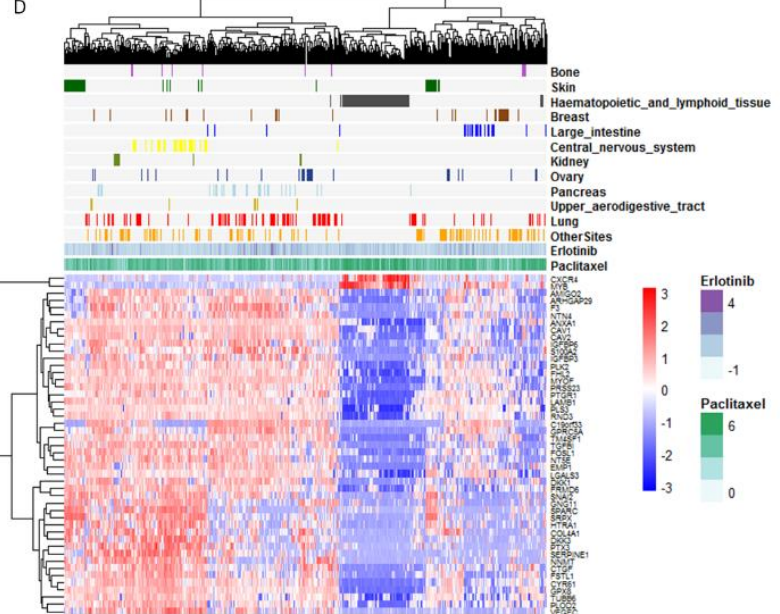

Figure 2. Hubs in a pan-cancer transcriptomic network display drug sensitivity predictive potential. A. Snapshot of a (fully connected) weighted gene correlation network. Nodes and 
edges representing genes and their correlations respectively. Network hubs and non-hubs are colored in red and white respectively. A zoom-in view of examples of hub and non-hub nodes. The color intensity of edges reflect the expression correlations between such nodes and others in the network. B. Graphical summary of (non-redundant) Gene Ontology terms statistically over-represented in the list of 47 hub genes. Terms are projected onto a semantic similarity space with REViGO (Supek et al., 2011), in which similar terms are positioned closer to each other. Each term is represented by a bubble with color and size indicating the term's level of statistical enrichment in our list and frequency in the GO database respectively. C. Comparison of hubs vs. non-hubs on the basis of their individual associations with drug sensitivity. The boxplot depicts the mean correlation between the gene expression and the AA values across CCLE cell lines. Box notches indicate $95 \%$ confidence interval for each median value. D. Cell line-drug experiments are visualized in terms of the 47-gene expression data. The panel above the gene expression heatmap illustrates the AA values observed for selected sets of cancer cell lines (grouped by tissue site) and two compound examples (Erlotinib and Paclitaxel) for illustration purposes.

We identified network hubs by extracting those genes with statistically detectable connectivity scores (i.e., weighted degree values) using WiPer (Azuaje, 2014). This resulted in 47 hubs (WiPer adjusted-P $<0.05$, online resource and Figure $\mathrm{S} 1)$, one of which (ANAX1) is illustrated in Figure 2A together with an example of a non-hub node (HCLS1). The 47 hub genes are significantly implicated in a wide diversity of biological processes and pathways of relevance to cancer progression and therapeutic response. They include cell proliferation, death, migration, adhesion, angiogenesis, kinase signaling and the extracellular matrix (Figures 2B and S1). 
Next, we analyzed drug sensitivity data (activity areas, AA) associated with these cell lines (11670 cell line-drug experiments) available in the CCLE. The AA, which is inversely correlated with the IC50 (the drug concentration at which an inhibition of $50 \%$ of cell viability is achieved), was defined by the CCLE to approximate the efficacy and potency of a drug simultaneously (Barretina et al., 2012). We stress that such data were not considered during the network generation and analysis steps. For each gene in the network, we calculated the correlation between gene expression and AA across all available (cell line-drug) data, and observed that: a. hubs tend to be anti-correlated with drug sensitivity, and b. such an anticorrelation is significantly stronger than in the case of non-hub genes. Moreover, such an association is considerably different to that displayed by non-hubs (Figure 2C). The 47 hub genes did not include previously reported markers of drug sensitivity: $A L K, B R A F, E R B B 2$, EGFR, HGF, NQ01, MDM2, MET and VEGFRs (Barretina et al., 2012; Safikhani, 2017). To further demonstrate the potential relevance of these genes, we clustered the samples (available cell line-drug experiment data) based on their 47-gene (baseline) expression profiles and verified that these genes could, in principle, segregate samples according to cancer types (tissue sites) and highlight differential drug responses across samples (Figure 2D). Using an alternative visualization and (unsupervised) clustering technique (Figure S2), we verified the potential of these 47 genes' expression data to segregate samples in terms of their drug sensitivity. Overall, these results suggest that our 47 hubs represented a potentially novel, biologically-meaningful gene set with drug sensitivity prediction potential. 


\section{Predicting drug sensitivity based on the network-derived 47-gene expression profiles}

We used the expression values from the 47 network hubs and drug sensitivity data $(\mathrm{n}=$ 10981, cell line-drug experiments, i.e., samples, with full expression and AA data available) to generate a drug sensitivity prediction model based on multiple linear regression (Methods). For a given sample (47-gene expression profile) and drug (identity of one of the 24 CCLE drugs), the model estimates a sensitivity score that approximates the AA values observed in the CCLE. For model training and testing, we used separate datasets respectively through a 10 -fold cross-validation sampling procedure. Prediction capability was evaluated with multiple performance indicators that compare the predicted and observed sensitivity values: Pearson, Spearman and Kendall correlations, root-meansquared errors (RMSE) and a concordance index (Figure 3). 


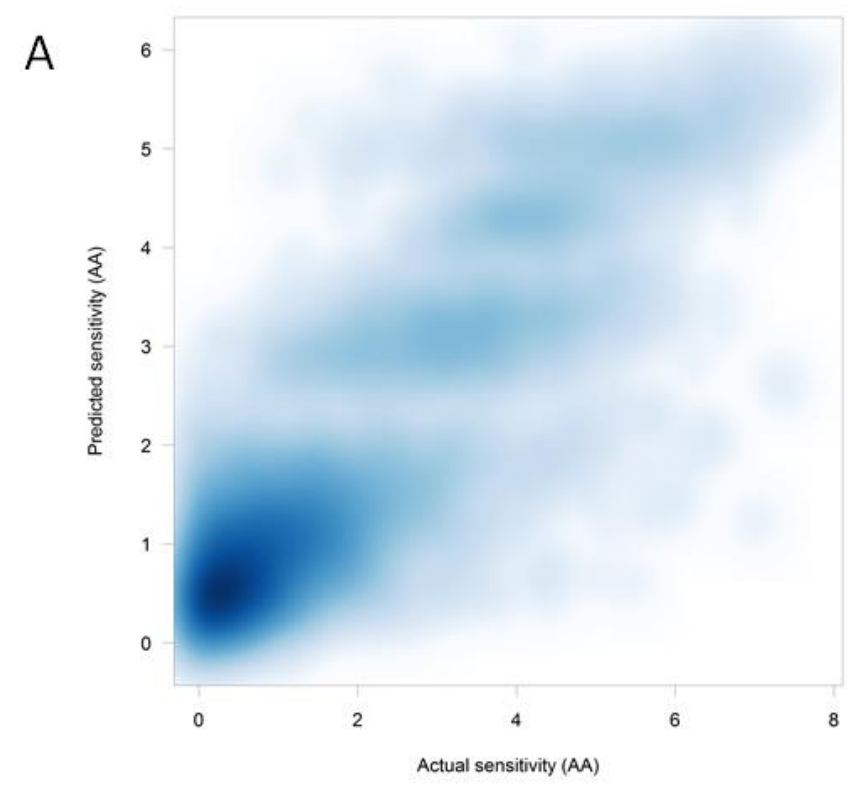

C
B

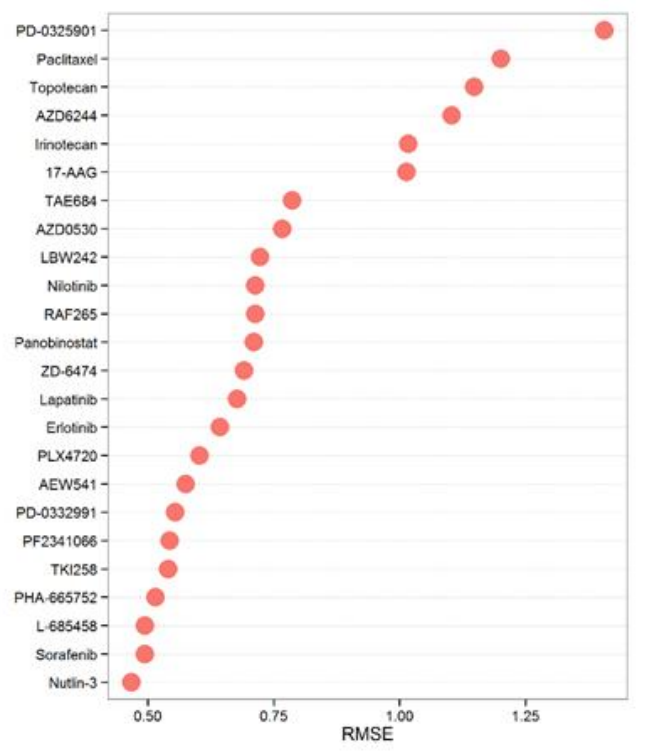

CCLE

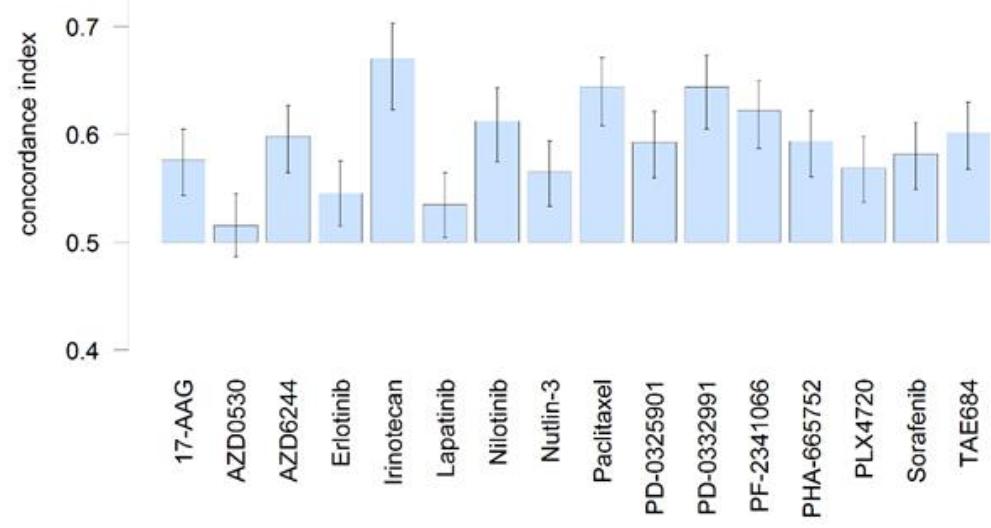

Figure 3. Alternative views of our model's predictive capacity on the CCLE dataset using alternative performance indicators. A. Density plot of predicted vs. actual sensitivity values $(n=10981)$. Pearson, Spearman and Kendall, correlations coefficients: $0.86,0.73$ and 0.54 respectively. B. Plot of root-mean-square errors (RMSE) observed for each drug. C. Concordance indices between the predicted and the observed AA values for a selected set of drugs. An index value $=0.5$ is the expected value from random prediction. Error bars: $95 \%$ confidence interval $(\mathrm{Cl})$ of the estimated concordance index. 
Figures $3 \mathrm{~A}$ and $\mathrm{S} 3$ show that the predicted and actual AA values are positively correlated (Pearson, Spearman and Kendall, correlations coefficients: $0.86,0.73$ and 0.54 respectively), which corroborates the predictive potential of our model. Such performance measures are comparable to, and in many cases outperform, those obtained from other published models trained and cross-validated on the same dataset. For example, a comprehensive analysis of different machine learning techniques (Jang et al., 2014), e.g., multiple-linear regression techniques, support vector machines and random forests, displayed (median) Pearson correlation coefficients falling into the range from 0.4 to 0.6 , including top-performing models generated with gene expression data or their combination with other data types (Jang et al., 2014).

Figure 3B offers an alternative assessment of our model's prediction capability based on the RMSE obtained for each CCLE drug. This plot offers two key insights: 1 . There are drugs for which our model can make relatively very accurate sensitivity predictions (e.g., Nutlin-3, an inhibitor of p53-Mdm2 complexes, and Sorafenib, a muti-kinase inhibitor) in comparison to other drugs (e.g., PD-0325901, a MEK inhibitor, and Paclitaxel, a mitotic inhibitor). 2. Our model's (drug-specific) prediction performance is competitive in relation to other published approaches trained and tested on the same dataset. For example, our model made predictions with a median RMSE $=0.70$ (range: $[0.47,1.40]$ ), which compares well with topperforming machine learning models that have reported median and minimum RMSE values above 0.80 and 0.65 respectively (Neto et al., 2014). For drugs such as Sorafenib, Nutlin-3 and PHA-665752, Dr.Paso tends to outperform models based on elastic-net and other variations of multiple-linear regression (Neto et al., 2014). Conversely, such models tend to offer relatively more accurate predictions for drugs such as Irinotecan and PD-0325901. These results corroborate previous findings about the lack of generalized solutions for highly 
accurately predicting sensitivity across all types of drugs (Fersini et al., 2014; Haverty et al., 2016; Jang et al., 2014).

To provide further insights into our model's prediction capacity, Figure $3 \mathrm{C}$ displays the concordance index for a selected set of drugs. For a random pair of samples, the concordance index estimates the probability of correctly predicting the relative sensitivities of such samples (e.g., sample $\mathrm{X}$ is more sensitive than sample $\mathrm{Y}$ ) in relation to the actual observed relative sensitivities. Perfect and random prediction performances are indicated by concordance indices equal to 1 and 0.5 respectively. Our model reported concordance indices with median values above 0.5 , which compares favorably with the results obtained by (Papillon-Cavanagh et al., 2013) with different alternative models, including multiple linear regression with elastic net, and applied to the same dataset. For instance, PapillonCavanagh et al. obtained concordance indices lower than 0.7 , including predictions with concordance indices falling below 0.5 for different drugs (e.g., Nutlin-3 and TAE684). These results suggest that our model can accurately predict drug sensitivity and provide, in relation to previously published models, promising predictive capability that we further investigated as follows.

\section{Model evaluation on an independent dataset}

We tested our 47-gene sensitivity prediction model on the 2016 release of the GDSC dataset (lorio et al., 2016a). To allow our CCLE-derived model to make predictions on this dataset, we focused on the 16 drugs that are found in both datasets. First, as in the case of the CCLE data, we show that the (baseline) expression profiles of the 47 genes can, in principle, cluster the GDSC samples according to cancer types (tissue sites) and highlight differential 
drug responses across samples (Figure 4A) in an unsupervised manner. Note that in the GDSC dataset drug sensitivity is represented as the logarithm of IC50 (LNIC50) values (AA values were not provided in this dataset). Using an alternative visualization and (unsupervised) clustering technique (Figure S2), we verified the potential of these 47 genes' expression data to segregate GDSC cell line-drug samples in terms of drug sensitivity.

Next, we applied our (CCLE-derived) prediction model to the GDSC data and made sensitivity predictions (AA values) for all the samples (cell line-drug experiments) available (Methods). The resulting predictions were then compared with the actual sensitivity values in the GDSC dataset (Figures 4B and S3). The predicted (AA) and actual sensitivity (LNIC50) values for these samples $(n=9984)$ are anti-correlated (Pearson, Spearman and Kendall, correlations coefficients: $-0.72,-0.71$ and -0.50 respectively). This indicates that our model is, in general, estimating sensitivity values that are in agreement with those observed in the test dataset, i.e., higher predictive agreement is reached when high AA (prediction) relates to a low LNIC50 (actual) values, and vice versa. 


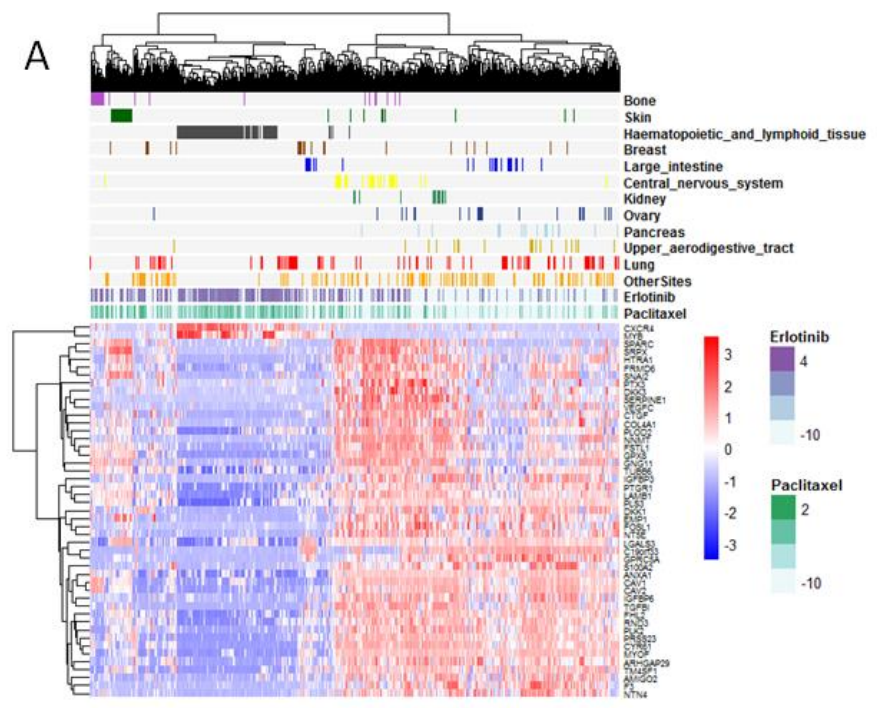

C

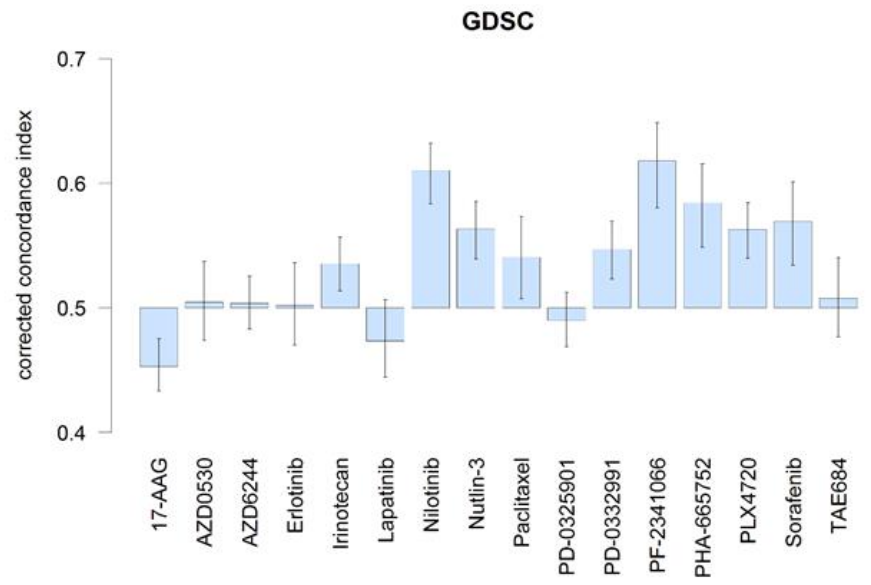

B

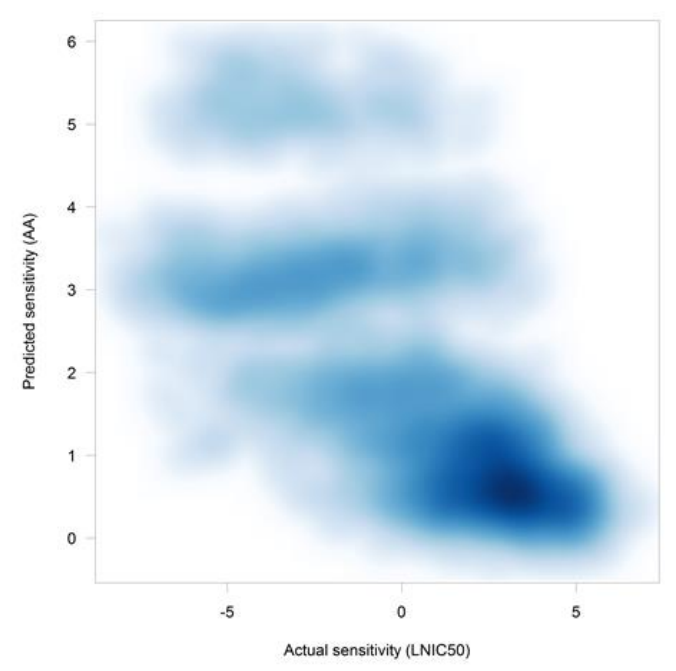

Figure 4. Alternative views of our model's prediction capacity on the GDSC dataset. A. Cell line-drug experiments are visualized in terms of the 47-gene expression data. The panel above the gene expression heatmap illustrates the LNIC50 $(\mu \mathrm{M})$ values observed for selected sets of cancer cell lines (grouped by tissue site) and two compounds (Erlotinib and Paclitaxel). B. Application of CCLE-derived model to the GDSC data. Density plot of predicted (AA) vs. actual sensitivity (LNIC50) values for drugs that are common between the CCLE and GDSC ( $\mathrm{n}=9984)$. Pearson, Spearman and Kendall, correlations coefficients: $-0.72,-0.71$ and -0.50 respectively. C. Concordance indices between the predicted and the observed sensitivity values. An index value $=0.5$ is the expected value from random prediction. Indices are corrected to account for the notion that higher concordance is 
reached when high AA (prediction) corresponds to a low LNIC50 (observed) values, and vice versa. Error bars: $95 \%$ confidence interval $(\mathrm{Cl})$ of the estimated concordance index.

Figure 4C summarizes the assessment of our model's predictive performance on the GDSC dataset based on drug-specific concordance indices, as done for the CCLE dataset (Figure 3). Concordance indices $>0.5$ were obtained for twelve out of the 16 drugs, and (among those 12 drugs) concordance estimates for 9 drugs can be reliably interpreted as larger than 0.5 (95\% confidence intervals of the estimated indices). The predictive performances for several drugs (e.g., Nilotinib, Nutlin-3 and Sorafenib) are very similar to those estimated in the CCLE dataset. As in the CCLE dataset, the sensitivity observed in samples treated with AZD0530 and Lapatinib proved to be more difficult to accurately to predict. Although concordance indices $>0.5$ were obtained for Irinotecan and Paclitaxel predictions, this represented a reduction of prediction performance in comparison to the predictions made for CCLE samples. The prediction performance of 17-AAG, PD-0325901 and TAE684 were also diminished. A previous study, which also used the GDSC dataset, consistently reported concordance indices $<0.5$ for Sorafenib (Papillon-Cavanagh et al., 2013). Moreover, in comparison to that study's models, our model reported comparable or higher concordance indices for other drugs, such as Nilotinib and PF-2341066 (Crizotinib). Conversely, such a previous study reported better prediction performances for 17-AAG, Lapatinib and PD0325901. Such comparisons should, nevertheless, be interpreted with caution as PapillonCavanagh et al.'s concordance indices were obtained with an older version of the GDSC dataset, which was used for both model training and testing. Overall, our findings further corroborate the predictive potential of our model, and highlight strengths and challenges in a drug-specific context. 


\section{Independent in vitro validation on several cell lines and compounds}

To further validate our model's predictive capability on independently-generated data, we generated predictions and performed in vitro tests for several GBM cell lines and compounds. First, we measured the (baseline) expression profiles of 4 GBM cell lines that have been well-characterized in our lab: U87, NCH644, NCH601 and NCH421k (Methods). While the CCLE and GDSC datasets included U87, the latter three are stem-like GBM cell lines that were not included in the previous model training and test phases.

Although genome-wide expression (microarray) data can appropriately cluster multiple samples (biological replicates) from such cell lines, we found that the expression profiles of our model's 47 genes are sufficient to achieve the same biologically-meaningful segregation while offering a clearer, fine-grained view of their differences (Figure S4). We also verified the platform-independent replicability of these results with another 47-gene expression dataset derived from 3 of these cell lines measured with qPCR (Figure S4). These results corroborate the biologically-relevant discriminatory capacity and reproducibility of our model's 47-gene expression patterns.

Next, our model predicted the sensitivity of our 4 GBM cell lines (18 samples in total, Methods) against the 24 drugs included in our model. The 47-gene (microarray) expression profiles of these cells were input to the prediction model ( $6 \mathrm{U} 87,3 \mathrm{NCH} 644,3 \mathrm{NCH} 601$ and $6 \mathrm{NCH} 421 \mathrm{k}$ gene expression profiles). Figure 5A summarizes the 432 predicted sensitivity (AA) values according to drug (18 predictions per drug). To investigate such predictions in vitro, we focused on the top-3 drugs associated with the highest predicted sensitivities (Paclitaxel, Panobinostat and 17-AAG), as well as on Erlotinib, which was predicted as a 
relatively ineffective compound. These drugs correspond to four different drug classes: cytotoxic, histone deacetylase inhibitor, antibiotic derivative and an EGFR inhibitor respectively. In the case of Erlotinib, the predictions are consistent with the fact that the tested cells do not (NCH644, NCH421k) or very lowly (U87, NCH601) express EGFR. The Figures 5B and S5 show a more focused view of the predicted sensitivity values for our samples against these 4 drugs.
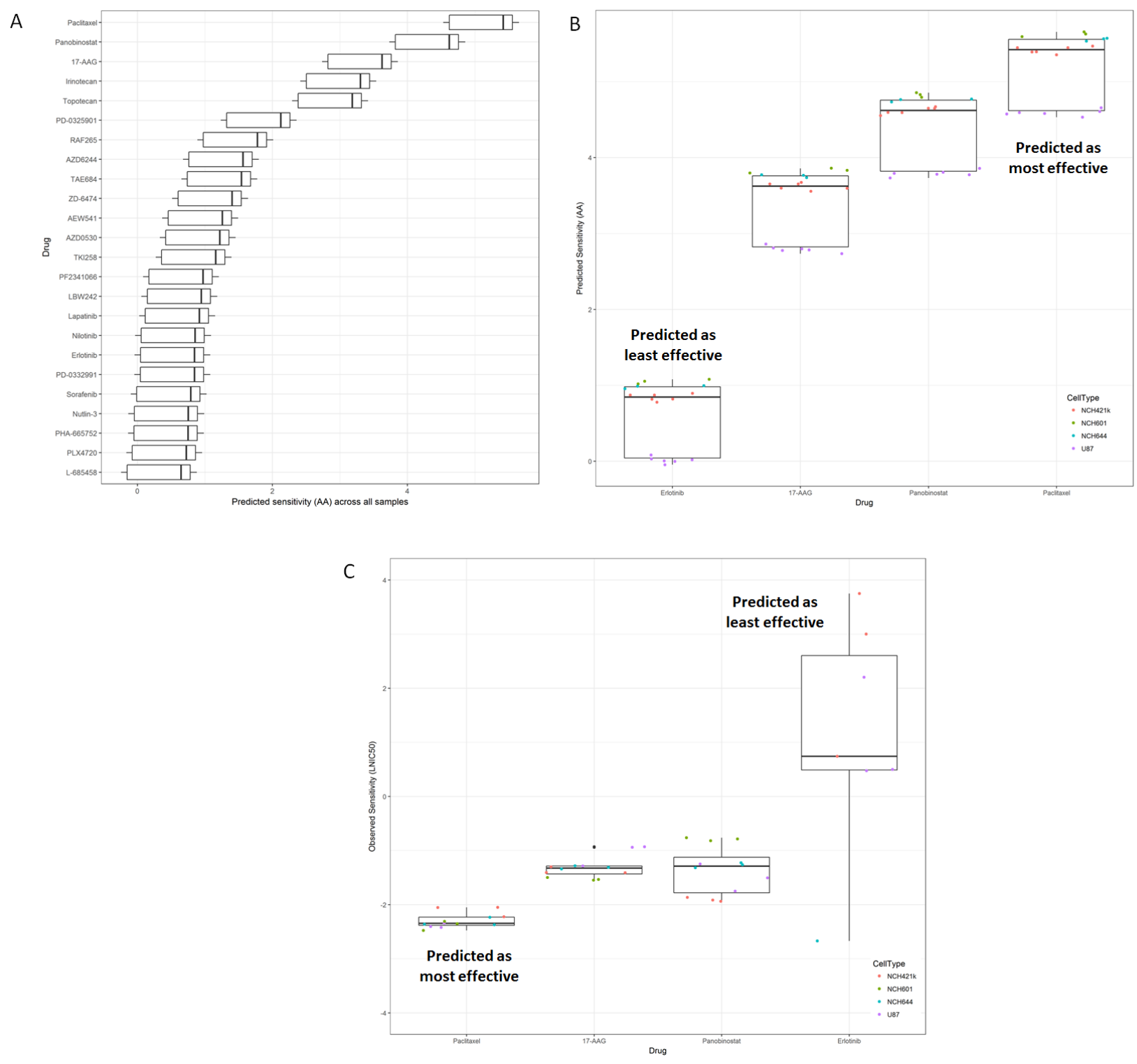

Figure 5. Drug sensitivity predictions and in vitro validation for different glioblastoma cell lines and compounds. A. Sensitivity predictions (horizontal axis) for 24 drugs (vertical axis). Box plot summarizes the (432) predicted sensitivity (AA, as defined in the prediction model) 
values for 4 glioblastoma cell lines: U87, NCH644, NCH601 and NCH421k. The 47-gene expression profiles of multiple biological replicates (18 samples in total) were input to the prediction model (6 U87, $3 \mathrm{NCH} 644,3 \mathrm{NCH} 601$ and $6 \mathrm{NCH} 421 \mathrm{k}$ samples). B. Alternative boxplot summary of the prediction results for 4 drugs (Erlotinib, 17-AAG, Panobinostat and Paclitaxel) and the different cell lines. These drugs, which were selected for subsequent in vitro tests, were predicted to be relatively highly (17-AAG, Panobinostat and Paclitaxel) and lowly (Erlotinib) effective against the 4 cell lines. C. Summary of in vitro test results. The selected drugs were tested on each cell line in triplicates, relative viability (vs. vehicle-treated samples) was measured for 8 drug concentration values $(\mu \mathrm{M})$ and IC50 values were estimated for each drug-sample experiment. The boxplot shows the resulting LNIC50 values obtained. Drug response data for NCH601 samples and Erlotinib are not available, and for NCH644 samples and Erlotinib not shown because of lack of effect. Boxes show the median, the $25^{\text {th }}$ and $75^{\text {th }}$ percentiles (lower and upper hinges), and (1.5 $\left.x\right)$ inter-quartile ranges.

We tested the selected drugs on each cell line, in triplicates, and measured their response based on their relative viability (i.e., normalized to vehicle-treated samples) for 8 drug concentration values $(\mu \mathrm{M})$. For each treated cell line, we estimated the IC50 values and compared them on the basis of cell line and drug groups. Figure $5 \mathrm{C}$ summarizes the results with boxplots showing the LNIC50 values. Drug response data for $\mathrm{NCH} 601$ samples and Erlotinib were not available (not tested), and data for NCH644 samples and Erlotinib are not shown due to lack of effect. Figure S6 includes all the drug response curves and additional details.

As predicted by our model, all our cell lines exhibited the lowest sensitivity, i.e., the highest IC50 values, when treated with Erlotinib (median LNIC50 $=0.74 \mu \mathrm{M}$ ). U87 was the least 
sensitive cell line in relation to all 4 drugs (median LNIC50 $=-1.27 \mu \mathrm{M}$ across all sampledrug experiments), in full agreement with the predictions. Our model consistently predicted $\mathrm{NCH} 601$ as the most sensitive cell line against all drugs (Figures S6). Our in vitro tests showed that $\mathrm{NCH} 421 \mathrm{k}$ tended to be more sensitive than $\mathrm{NCH601}$ (median loglC50: -1.64 vs. $-1.54 \mu \mathrm{M})$. Despite this particular discrepancy, we found global agreement between predicted and observed sensitivities on the basis of cell type (Spearman correlation between the median sensitivity values, predicted (AA) vs. observed (LNIC50) in the 4 cell line groups: $-0.40)$

In accordance with the predictions, Paclitaxel was the most effective drug across all treated samples (median LNIC50 = -2.35 $\mu \mathrm{M}$ ). Lesser agreement between predicted and observed sensitivities were obtained in the case of the remaining two drugs. For all samples, our model predicted overall higher sensitivity for Panobinostat than for 17-AAG (Figure 5B). In vitro, relatively similar responses were obtained for Panobinostat (median LNIC50 $=-1.29$ ) and 17-AAG (median LNIC50 $=-1.33 \mu \mathrm{M}$ ), though a larger variability of sensitivity was observed in the former case. Nevertheless, predictions and in vitro tests concordantly showed that $\mathrm{NCH} 421 \mathrm{k}$ and U87 samples treated with Panobinostat were consistently more sensitive than all samples treated with 17-AAG (Figures 5C and S6). Taken together, these results provide further evidence of the predictive capacity of our model. The resulting system, Dr.Paso, will enable the community to conduct further investigations.

\section{Dr.Paso online}

To share our model and enable further research, we developed a web-accessible tool that allows researchers to upload their own gene expression data, make sensitivity predictions 
and visualize results in a few steps (Figure 6). The Help section of the website offers a guided application example using CCLE data. Users provide their input data as a text file containing the (baseline) 47-gene expression for different samples, and then can select all or specific drugs for making predictions (Figure 6A). Dataset re-scaling (feature standardization with means and standard deviations equal to 0 and 1 respectively) can be applied to harmonize the input dataset with the feature representation used in our model. Prediction results are presented with graphical displays and tables in different panels. Moreover, users can control the amount and focus of information at the drug and sample levels (Figure 6B to 6D). Results can be saved in different graphical and tabular file formats. The tool is freely available at www.drpaso.lu.

A

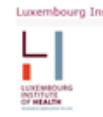

Dr.Paso Drug response prediction and analysis
system for oncology research
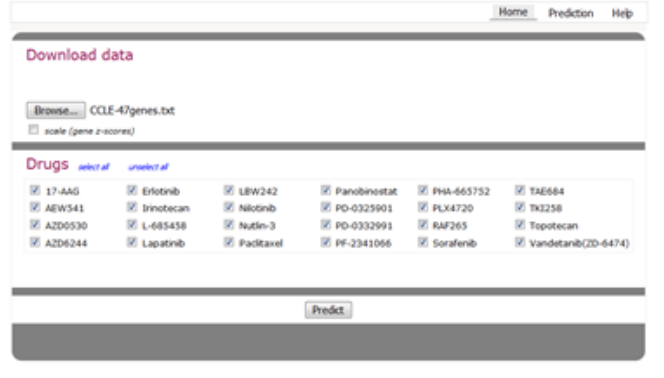

c 닐

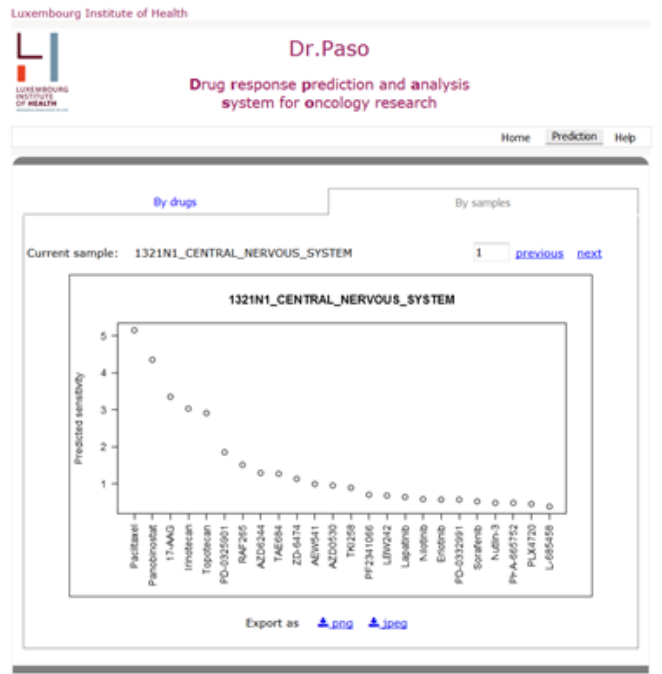

B

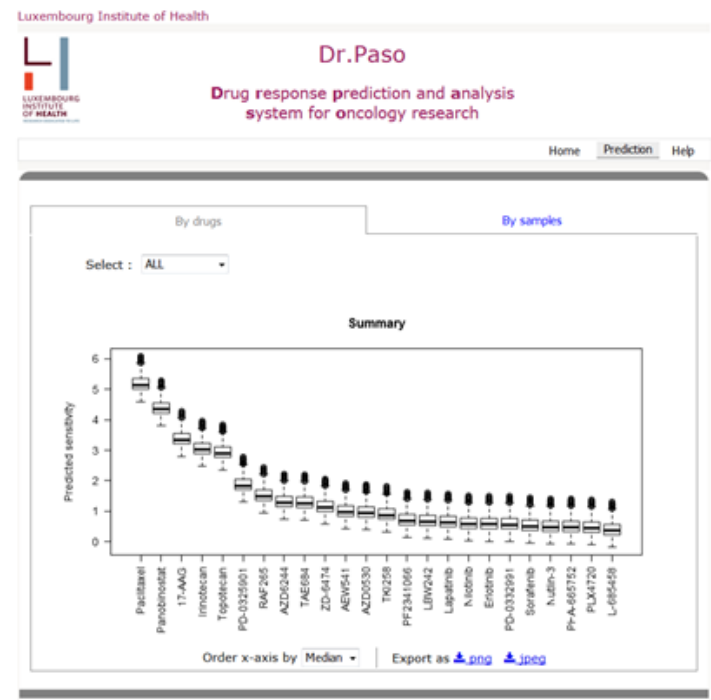

D

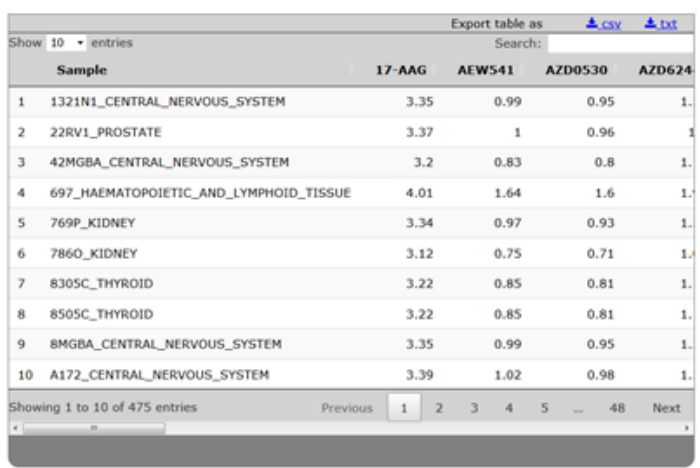


Figure 6. Dr.Paso online: a Web-based tool for predicting drug sensitivity and enabling further research. Screenshots of: A. Main page with user input and analysis options; B: Global view of predicted sensitivity values for a given input gene expression dataset and all drugs available in the CCLE; C: Alternative view of predictions focused on a specific input sample and all drugs; D. Tabular-based view of results. All views can be selected and downloaded according to user requirements.

\section{Discussion}

The development of computational models for estimating drug sensitivity based on the analysis of large and diverse collections of cancer cell lines is important to support preclinical research, and provides a basis for future clinically-oriented applications. Access to such models and their user-friendly application will enable new research across oncology domains and additional computational investigations. Our Drug Response Prediction and Analysis System for Oncology research, Dr.Paso, addresses such needs through the integration of network-based and statistical modeling approaches. For a given drug, our system predicts an anti-cancer sensitivity score based on the gene expression profile of 47 genes, which were shown to represent hubs in a pan-cancer transcriptomic network and to be prominently implicated in a variety of cancer-relevant biological processes. Dr.Paso was generated and evaluated on independent datasets, including our own in vitro validations of several cell line-compound combinations, and showed promising results in terms of predictive accuracy and concordance. Future research can apply Dr.Paso to 47-gene expression signatures from patient samples to investigate its potential relevance in the clinical setting. 
Our study and other previous research highlight the challenges faced and complementary predictive capacity exhibited by different modeling approaches (Costello et al., 2014; Papillon-Cavanagh et al., 2013). No single model can consistently make accurate predictions for all drugs and cell lines available in the CCLE and GDSC datasets, including models that include genomic data (Gupta et al., 2016; Jang et al., 2014; Menden et al., 2013). Different models can offer more, or less, accurate predictions for certain drugs, and there is no conclusive evidence about the dominance of a particular modeling technique (Azuaje, 2017). Such limitations may be partially explained by a lack of sufficient molecular information to account for the complexity of cell lines and their drug responses, choice of surrogate measures of drug sensitivity and inconsistencies of sensitivity data between the CCLE and GDSC (Haverty et al., 2016; Investigators, 2015; Safikhani, 2017). The latter may also partly explain the overall degradation of predictive performance when training models on the CCLE and testing them on the GDSC.

Dr.Paso generates sensitivity scores based on a multiple linear regression model. We, as others elsewhere, have shown that relatively less complex regression models can offer comparable, and in some cases better, prediction performance than those models based on larger sets of learning parameters. Dr.Paso's predictive capacity is grounded in an unbiased network-guided selection of model inputs (47 genes) prior to the fitting of the regression model. Such a discovery process was shown to be both statistically- and biologicallymeaningful. Apart from our multiple linear regression, we applied other regression techniques, e.g., support vector machines and neural networks, but decided to focus our investigation on a model with relatively lower complexity. Collectively, Dr.Paso is based on a biomarker discovery and prediction-making methodology that is both biologically-driven and statistically-powerful. New investigations, motivated by new datasets and clinically- 
oriented questions, are certainly envisaged and are expected to include new biomarker discovery and prediction modeling strategies.

Notwithstanding recent advances in the field, there is a need to make executable models accessible to the research community to enable new investigations, including new applications and comparative analyses among different techniques. Here we offer Dr.Paso as a publicly-accessible online tool. While further investigations are needed, our study offers further evidence of the potential of computational models for predicting anti-cancer sensitivity. In the short-term, our findings will enable new pre-clinical research applications and may provide a new perspective for bringing such models closer to the clinic.

\section{Methods}

\section{Identification of $\mathbf{4 7}$ genes with drug sensitivity predictive potential}

The published pre-processed CCLE (microarray) gene expression and drug sensitivity datasets were obtained from the CCLE website. In the gene expression dataset, we focused on genes with symbols, calculated their standard deviation (SD) across all samples (1037) and ranked them based on their SD. For further analyses, we selected the most variable genes: 177 genes with SD values above the $99^{\text {th }}$ percentile of the SD value distribution. We computed the gene-gene (Pearson) correlation coefficients between all the 177 genes and merged them into a single gene expression correlation network. We applied WiPer (Azuaje, 2014) to this fully-connected weighted network to detect highly connected nodes (hub genes). For each network node, WiPer computes the weighted degree and a corresponding P-value to assess the significance of the observed values, and adjusts it for multiple testing. 
Genes exhibiting (Bonferroni adjusted) $\mathrm{P}<0.05$ were considered hubs (47 genes). Drug sensitivity information was not used to select hubs. The resulting 47 genes were examined with different Gene Ontology (GO) and biological pathway analysis tools (below). For each hub gene, we estimated the correlation of its expression profile (across all samples) with the activity area $(A A)$ values available from all sample-drug combinations. The AA was formulated by the CCLE to approximate the efficacy and potency of a drug simultaneously and is inversely correlated with the IC50 (Barretina et al., 2012). We compared hubs and non-hubs on the basis of such individual expression-sensitivity correlations. Visualizations and unsupervised clustering of hubs and cell lines described by hub expression values were implemented with different open-source tools (below).

\section{Training and testing of prediction model}

We represented each CCLE sample (cell line-drug combination) with the expression values of the 47 hub genes and their corresponding AA values. We focused on samples with complete expression and AA data. The resulting set of 10981 samples was used for training and testing regression models. The dataset was standardized by re-scaling each gene so that each gene has mean and standard deviation of 0 and 1 respectively. For each model, we implemented 10-fold cross-validation (CV) for separating training from testing and for assessing prediction performance. We also used leave-one-out CV (LOOCV) and similar prediction performance results were obtained. Diverse regression techniques with different levels of complexity were investigated. We focused on a multiple linear regression model with Ridge regularization (Ridge parameter $=1 \mathrm{E}-08$ ) because its performance (regression errors) was better than or comparable to those obtained with other techniques, such as support vector machines and k-nearest neighbors, and because of its interpretability in 
comparison to relatively more complex models. The accuracy of model predictions was assessed by measuring their (Pearson, Spearman and Kendall) correlations with the observed values in the CCLE, the root-mean-squared error (RMSE) and a concordance index. The latter approximates, for a random pair of samples, the probability of correctly predicting which sample is more (or less) sensitivity than the other (Harrell et al., 1996). A concordance index equal to 0.5 indicates that the model's performance is comparable to that from a random predictor, while an index equal to 1 represents the perfect predictor.

\section{Independent evaluation on the GDSC dataset}

Raw expression data were obtained from the ArrayExpress database (accession number EMTAB-3610) and drug sensitivity (natural logarithm of the IC50 in $\mu M$, LNIC50) were downloaded from GDSC database (http://www.cancerrxgene.org, release-5.0). We normalized raw expression data with the RMA function of R/oligo package (Carvalho and Irizarry, 2010). Then we averaged the resulting log2 probe-set intensities to estimate the expression of each gene. Associations between probe-sets and gene symbols were obtained through the hgu219.db annotation package (Carlson, 2016). For each cell linedrug experiment available (sample), we retrieved the expression data for the 47 genes used as inputs to our prediction model and retrieved the corresponding drug sensitivity. We focused on the 16 drugs found in both this and the CCLE dataset. This resulted in a dataset consisting of 9984 samples, each one represented by 47 gene expression values and one LNIC50 value. We standardized expression data as in the case of the CCLE dataset, reformatted the file and input it to the CCLE-derived prediction model (further information below). For each sample in the dataset, the model predicted a drug sensitivity score (approximation of AA). We compared predicted vs. observed values using the indicators 
applied to the CCLE dataset analysis. We adapted the concordance index to account for the fact that AA and LNIC50 are expected to be anti-correlated, i.e., for a given sample, concordance is achieved when a high (predicted) AA value corresponds to a low (observed) LNIC50 value, and vice versa.

\section{GBM cell lines and expression data for in vitro validations}

U87 cells were obtained from the ATCC (Rockville, USA) and were cultured as monolayers in DMEM containing 10\% FBS, 2 mM L-Glutamine and $100 \mathrm{U} / \mathrm{ml}$ Pen-Strep (Lonza). GBM stem-like cultures ( $\mathrm{NCH} 421 \mathrm{k}, \mathrm{NCH} 601$ and $\mathrm{NCH} 644)$ were kindly provided by Christel Herold-Mende (University of Heidelberg, Germany) and were cultured as 3D non-adherent spheres as previously described (Abdul Rahim et al., 2017; Sanzey et al., 2015).

We measured the (baseline) gene expression of 4 GBM cell lines using microarrays (6 U87, $6 \mathrm{NCH} 421 \mathrm{k}, 3 \mathrm{NCH} 644$ and $3 \mathrm{NCH} 601$ biological replicates), as reported in (Sanzey et al., 2015). For our model's 47 genes, we also replicated gene expression measurements using qPCR for U87, NCH421k and NCH644 cell lines (each one in triplicate). RNA was extracted from $10^{6}$ cells using TRI Reagent® (Sigma-Aldrich). RNA isolated in the aqueous phase with a Phase lock gel-Heavy (5 Prime) was precipitated with 100\% isopropanol and purified using RNeasy ${ }^{\circledR}$ Mini kit combined with an on-column DNase treatment (Qiagen). For the qPCR, RNA was reverse-transcribed into cDNA using Superscript III ${ }^{\mathrm{TM}}$ (Invitrogen) following manufacturer's instructions. qPCR was performed in 96-well plates using SYBRß Green Master Mix (Bio-Rad) and CFX-96 thermal cycler (Bio-Rad). Normalized gene expression levels were calculated using the CFX manager 3.1 software (Bio-Rad) via the delta-delta Cq method with "Hspcb, Rps13, 18sRNA" as reference genes and taking into account the 
calculated amplification efficiency for each primers pair. We provide a MIQE-compliance checklist table as a supplemental item.

\section{Drug sensitivity predictions and in vitro validation on GBM cell lines}

The gene (microarray) expression dataset was standardized as above. Each sample, represented by a 47-gene (microarray) expression profile, was input to the prediction model and a drug sensitivity value was predicted for each one of them (18 samples in total), for each of the 24 drugs included in the model. Predicted values were compared between them to determine their relative differences in terms of cell lines and drugs. Next, these predictions were compared to the in vitro sensitivity values that were obtained as follows. We tested 4 drugs: Paclitaxel (Sigma-Aldrich), Panobinostat, 17-AAG and Erlotinib (Selleck Chemicals) independently on the selected 4 GBM cell lines with 8 drug concentrations. For each cell line and dose, we performed treatment experiments in triplicate (i.e., 3 treated biological replicates / dose). As a measurement of drug sensitivity, WST-1 (Sigma-Aldrich) cell viability assays were implemented. U87, $\mathrm{NCH} 421 \mathrm{k}, \mathrm{NCH} 644$ and $\mathrm{NCH} 601$ cell lines were seeded into 96-well plates at densities of $1,500,5,000,4,000,6,000$ cells per well, in appropriate culture medium (Sanzey et al., 2015). Cells were incubated, 24h hours after seeding, with the 8 different drug concentrations ranging from $10 \mu \mathrm{M}$ to $6.1 \times 10-4 \mu \mathrm{M}$, with a final volume of DMSO not exceeding $0.1 \%$ and each condition was tested with 6 technical replicates. After $72 \mathrm{~h}$ incubation, WST-1 reagent was added in medium to a final concentration of $10 \%$. Adherent cell line (U87) was incubated at $37^{\circ} \mathrm{C}$ for 2 hours and $3 \mathrm{D}$ sphere stem-like cell lines ( $\mathrm{NCH} 421 \mathrm{k}, \mathrm{NCH} 644$ and $\mathrm{NCH} 601)$ were incubated at $37^{\circ} \mathrm{C}$ for $6-8$ hours. Absorbance was measured against a background control at 450nm on a FLUOstar OPTIMA Microplate Reader (BMG LABTECH). Using the normalized viability measurements, we generated drug 
dose-response curves and estimated IC50 values $(\mu \mathrm{M})$ for each sample-drug combination. The dose-response curves were fitted with a four-parameter logistic regression model, whose parameters were calculated using GraphPad Prism 7 (GraphPad).

\section{Software and Dr.Paso's Web-based tool}

We used the $\mathrm{R}$ statistical environment for data analysis and visualization (www.rproject.org), packages: ggplot2, pheatmap, MASS and SNFtool (Wang et al., 2014). Concordance indexes (Harrell et al., 1996) were calculated based on rescaled Kendall rank correlation coefficients, which were also used to estimate confidence intervals (by Fisher's transformation). For network analyses, we applied Cytoscape for visualization (Shannon et al., 2003), MINE for similarity exploration (Reshef et al., 2011) and WiPer for network hub identification (Azuaje, 2014). REViGO (Supek et al., 2011) and g:Profiler (Reimand et al., 2007) were applied for biological process and pathway enrichment analyses. The Weka workbench was used for building and testing regression models (Frank, 2016; Hall, 2009), and GraphPad Prism (www.graphpad.com) for analyzing drug response curves. We provide researchers with a Web-based application to enable them to predict anticancer drug sensitivity using their own (47-gene) transcriptomic data. The tool is based on the R/Shiny package (https://shiny.rstudio.com/). Although this package offers useful functionality for generating an interactive user interface, we customized available code using the R/Shinyjs package (http://deanattali.com/shinyjs/). Users can input pre-processed expression datasets. Alternatively, our application can also implement z-score rescaling of the input data. Figures containing the prediction results can be downloaded and stored as either png or jpeg files. Results are also shown as tables with sample-specific predictions (in rows) with their corresponding drugs (in columns), and may be stored as either csv or tsv files. 


\section{Author Contributions}

Conceptualization, F.A.; Methodology, F.A., P.V.N, C.J., T.K., A.G, S.P.N; Software, F.A., T.K., P.V.N, C.J., A.M., S.K.; Validation, T.K, C.J.; Resources, A.G, G.D, S.P.N, Supervision, F.A., A.G., S.P.N, G.D.; Writing - Original Draft, F.A.; Writing - Review \& Editing, all authors.

\section{Acknowledgments}

This research was funded by (LIH-MESR) project Connect2Predict to F.A. For technical guidance to C.J, we thank H. Erasimus and S. Fritah (drug experiments) and V. Barthelemy, A. Bernard, J. Bohler and A. Dirkse (cell line manipulation) at the LIH NorLux NeuroOncology Laboratory. For helpful feedback on the manuscript, we thank L.C. Tranchevent at the LIH Proteome and Genome Research Unit.

\section{References}

Abdul Rahim, S.A., Dirkse, A., Oudin, A., Schuster, A., Bohler, J., Barthelemy, V., Muller, A., Vallar, L., Janji, B., Golebiewska, A., et al. (2017). Regulation of hypoxia-induced autophagy in glioblastoma involves ATG9A. British journal of cancer 117, 813-825.

Azuaje, F. (2017). Computational models for predicting drug responses in cancer research. Briefings in bioinformatics $18,820-829$.

Azuaje, F.J. (2014). Selecting biologically informative genes in co-expression networks with a centrality score. Biology direct 9, 12 .

Barretina, J., Caponigro, G., Stransky, N., Venkatesan, K., Margolin, A.A., Kim, S., Wilson, C.J., Lehar, J., Kryukov, G.V., Sonkin, D., et al. (2012). The Cancer Cell Line Encyclopedia enables predictive modelling of anticancer drug sensitivity. Nature 483, 603-607.

Caponigro, G., and Sellers, W.R. (2011). Advances in the preclinical testing of cancer therapeutic hypotheses. Nature reviews Drug discovery 10, 179-187.

Carlson, M. (2016). hgu219.db: Affymetrix Human Genome 219 Plate annotation data (chip hgu219). R package version 3.2.3.

Carvalho, B.S., and Irizarry, R.A. (2010). A framework for oligonucleotide microarray preprocessing. Bioinformatics (Oxford, England) 26, 2363-2367. 
Cortes-Ciriano, I., van Westen, G.J., Bouvier, G., Nilges, M., Overington, J.P., Bender, A., and Malliavin, T.E. (2016). Improved large-scale prediction of growth inhibition patterns using the NCI60 cancer cell line panel. Bioinformatics (Oxford, England) 32, 85-95.

Costello, J.C., Heiser, L.M., Georgii, E., Gonen, M., Menden, M.P., Wang, N.J., Bansal, M., Ammad-ud-din, M., Hintsanen, P., Khan, S.A., et al. (2014). A community effort to assess and improve drug sensitivity prediction algorithms. Nature biotechnology 32, 1202-1212.

Dong, Z., Zhang, N., Li, C., Wang, H., Fang, Y., Wang, J., and Zheng, X. (2015). Anticancer drug sensitivity prediction in cell lines from baseline gene expression through recursive feature selection. BMC cancer 15 , 489.

Fersini, E., Messina, E., and Archetti, F. (2014). A p-Median approach for predicting drug response in tumour cells. BMC bioinformatics 15, 353.

Frank, E.H., M.A; Witten, I.H. (2016). The WEKA Workbench. Online Appendix for "Data Mining: Practical Machine Learning Tools and Techniques", Fourth Edition edn (Morgan Kaufmann).

Garnett, M.J., Edelman, E.J., Heidorn, S.J., Greenman, C.D., Dastur, A., Lau, K.W., Greninger, P., Thompson, I.R., Luo, X., Soares, J., et al. (2012). Systematic identification of genomic markers of drug sensitivity in cancer cells. Nature $483,570-575$.

Geeleher, P., Cox, N.J., and Huang, R.S. (2014). Clinical drug response can be predicted using baseline gene expression levels and in vitro drug sensitivity in cell lines. Genome biology 15, R47.

Goodspeed, A., Heiser, L.M., Gray, J.W., and Costello, J.C. (2016). Tumor-Derived Cell Lines as Molecular Models of Cancer Pharmacogenomics. Molecular cancer research : MCR 14, 3-13.

Gupta, S., Chaudhary, K., Kumar, R., Gautam, A., Nanda, J.S., Dhanda, S.K., Brahmachari, S.K., and Raghava, G.P. (2016). Prioritization of anticancer drugs against a cancer using genomic features of cancer cells: A step towards personalized medicine. Scientific reports 6, 23857.

Hall, M., Frank, E., Holmes, G., Pfahringer, B., Reutemann, P.; Witten, I.H. (2009). The WEKA Data Mining Software: An Update. SIGKDD Explorations 11.

Harrell, F.E., Jr., Lee, K.L., and Mark, D.B. (1996). Multivariable prognostic models: issues in developing models, evaluating assumptions and adequacy, and measuring and reducing errors. Statistics in medicine 15, 361-387.

Haverty, P.M., Lin, E., Tan, J., Yu, Y., Lam, B., Lianoglou, S., Neve, R.M., Martin, S., Settleman, J., Yauch, R.L., et al. (2016). Reproducible pharmacogenomic profiling of cancer cell line panels. Nature 533, 333-337.

Investigators, T.C.C.L.E.a.G.o.D.S.i.C. (2015). Pharmacogenomic agreement between two cancer cell line data sets. Nature $528,84-87$.

Iorio, F., Knijnenburg, T.A., Vis, D.J., Bignell, G.R., Menden, M.P., Schubert, M., Aben, N., Goncalves, E., Barthorpe, S., Lightfoot, H., et al. (2016a). A Landscape of Pharmacogenomic Interactions in Cancer. Cell 166, 740-754.

Iorio, F., Knijnenburg, T.A., Vis, D.J., Bignell, G.R., Menden, M.P., Schubert, M., Aben, N., Goncalves, E., Barthorpe, S., Lightfoot, H., et al. (2016b). A Landscape of Pharmacogenomic Interactions in Cancer. Cell 166, 740-754.

Jang, I.S., Neto, E.C., Guinney, J., Friend, S.H., and Margolin, A.A. (2014). Systematic assessment of analytical methods for drug sensitivity prediction from cancer cell line data. Pacific Symposium on Biocomputing Pacific Symposium on Biocomputing, 63-74.

Menden, M.P., lorio, F., Garnett, M., McDermott, U., Benes, C.H., Ballester, P.J., and Saez-Rodriguez, J. (2013). Machine learning prediction of cancer cell sensitivity to drugs based on genomic and chemical properties. PloS one 8, e61318. 
Neto, E.C., Jang, I.S., Friend, S.H., and Margolin, A.A. (2014). The Stream algorithm: computationally efficient ridge-regression via Bayesian model averaging, and applications to pharmacogenomic prediction of cancer cell line sensitivity. Pacific Symposium on Biocomputing Pacific Symposium on Biocomputing, 27-38.

Papillon-Cavanagh, S., De Jay, N., Hachem, N., Olsen, C., Bontempi, G., Aerts, H.J., Quackenbush, J., and Haibe-Kains, B. (2013). Comparison and validation of genomic predictors for anticancer drug sensitivity. Journal of the American Medical Informatics Association : JAMIA 20, 597-602.

Rees, M.G., Seashore-Ludlow, B., Cheah, J.H., Adams, D.J., Price, E.V., Gill, S., Javaid, S., Coletti, M.E., Jones, V.L., Bodycombe, N.E., et al. (2016). Correlating chemical sensitivity and basal gene expression reveals mechanism of action. Nature chemical biology 12, 109-116.

Reimand, J., Kull, M., Peterson, H., Hansen, J., and Vilo, J. (2007). g:Profiler--a web-based toolset for functional profiling of gene lists from large-scale experiments. Nucleic acids research 35, W193-200.

Reinhold, W.C., Sunshine, M., Liu, H., Varma, S., Kohn, K.W., Morris, J., Doroshow, J., and Pommier, Y. (2012). CellMiner: a web-based suite of genomic and pharmacologic tools to explore transcript and drug patterns in the NCl-60 cell line set. Cancer research 72, 3499-3511.

Reshef, D.N., Reshef, Y.A., Finucane, H.K., Grossman, S.R., McVean, G., Turnbaugh, P.J., Lander, E.S., Mitzenmacher, M., and Sabeti, P.C. (2011). Detecting novel associations in large data sets. Science (New York, NY) $334,1518-1524$.

Ross, N.T., and Wilson, C.J. (2014). In vitro clinical trials: the future of cell-based profiling. Frontiers in pharmacology 5, 121.

Safikhani, Z.S., P; Freeman, M; El-Hachem, N; She, A; et al. Revisiting inconsistency in large pharmacogenomic studies (2017). Revisiting inconsistency in large pharmacogenomic studies. F1000Research 5

Sanzey, M., Abdul Rahim, S.A., Oudin, A., Dirkse, A., Kaoma, T., Vallar, L., Herold-Mende, C., Bjerkvig, R., Golebiewska, A., and Niclou, S.P. (2015). Comprehensive analysis of glycolytic enzymes as therapeutic targets in the treatment of glioblastoma. PloS one 10, e0123544.

Shannon, P., Markiel, A., Ozier, O., Baliga, N.S., Wang, J.T., Ramage, D., Amin, N., Schwikowski, B., and Ideker, T. (2003). Cytoscape: a software environment for integrated models of biomolecular interaction networks. Genome research 13, 2498-2504.

Stetson, L.C., Pearl, T., Chen, Y., and Barnholtz-Sloan, J.S. (2014). Computational identification of multi-omic correlates of anticancer therapeutic response. BMC genomics 15 Suppl 7, S2.

Supek, F., Bosnjak, M., Skunca, N., and Smuc, T. (2011). REVIGO summarizes and visualizes long lists of gene ontology terms. PloS one 6, e21800.

Wang, B., Mezlini, A.M., Demir, F., Fiume, M., Tu, Z., Brudno, M., Haibe-Kains, B., and Goldenberg, A. (2014). Similarity network fusion for aggregating data types on a genomic scale. Nature methods 11, 333-337.

Wilding, J.L., and Bodmer, W.F. (2014). Cancer cell lines for drug discovery and development. Cancer research 74, 2377-2384.

Yang, W., Soares, J., Greninger, P., Edelman, E.J., Lightfoot, H., Forbes, S., Bindal, N., Beare, D., Smith, J.A., Thompson, I.R., et al. (2013). Genomics of Drug Sensitivity in Cancer (GDSC): a resource for therapeutic biomarker discovery in cancer cells. Nucleic acids research 41, D955-961. 


\section{Supplemental Information}

\section{Dr.Paso:}

\section{Drug response prediction and analysis system for oncology research}

Francisco Azuaje ${ }^{1,+,}{ }^{*}$, Tony Kaoma², Peter V. Nazarov, Céline Jeanty, Arnaud Muller, Sang-Yoon Kim, Anna Golebiewska¹, Gunnar Dittmar², Simone P. Niclou.

${ }^{1}$ NorLux Neuro-Oncology Laboratory, Department of Oncology, Luxembourg Institute of Health (LIH), Luxembourg, Luxembourg.

2 Proteome and Genome Research Unit, Department of Oncology, Luxembourg Institute of Health (LIH), Luxembourg, Luxembourg. 

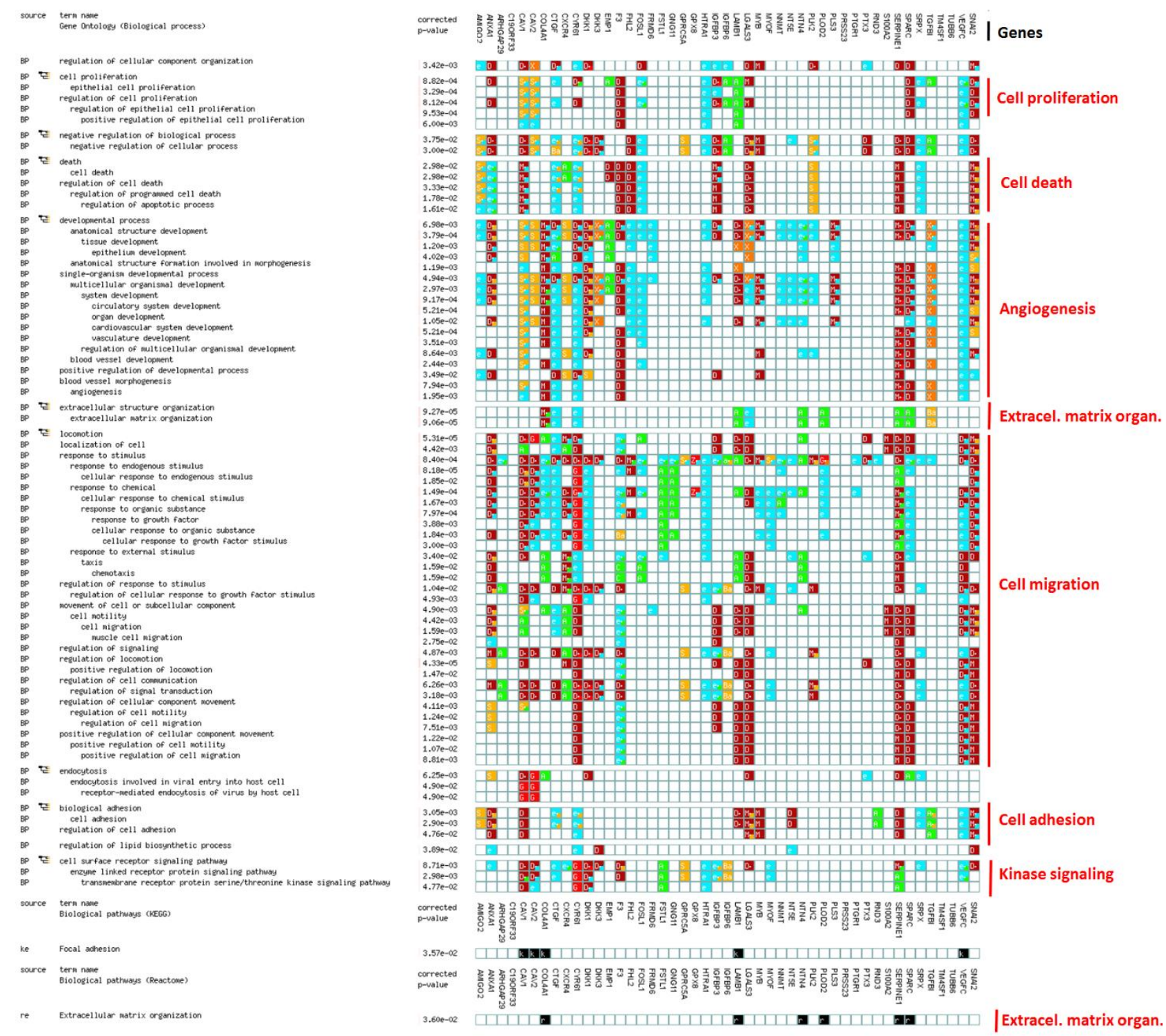

Figure S1. Statistical enrichment analysis of biological processes and pathways in the set of $\mathbf{4 7}$ network hubs. Related to Figure 2B. An alternative visualization of functional enrichments using G:Profiler (Reimand et al., 2007). As shown in Figure 2B, our set of 47 hub genes (columns) is significantly associated (at corrected P-value $=0.05$ ) with a diversity of biological processes and pathways (rows). Each colored cell represents the association between an individual gene and a functional annotation. Colors are used to specify the evidence type of the observed association: ${ }^{2}$ : Inferred from experiment, ${ }^{\mathrm{D}} \mathrm{IH}^{\mathrm{H}}$ : Direct assay / Mutant phenotype, ${ }^{\mathrm{B}}$ Traceable author, ${ }^{\mathrm{B}}$ : Electronic annotation, additional information at http://biit.cs.ut.ee/gprofiler. 

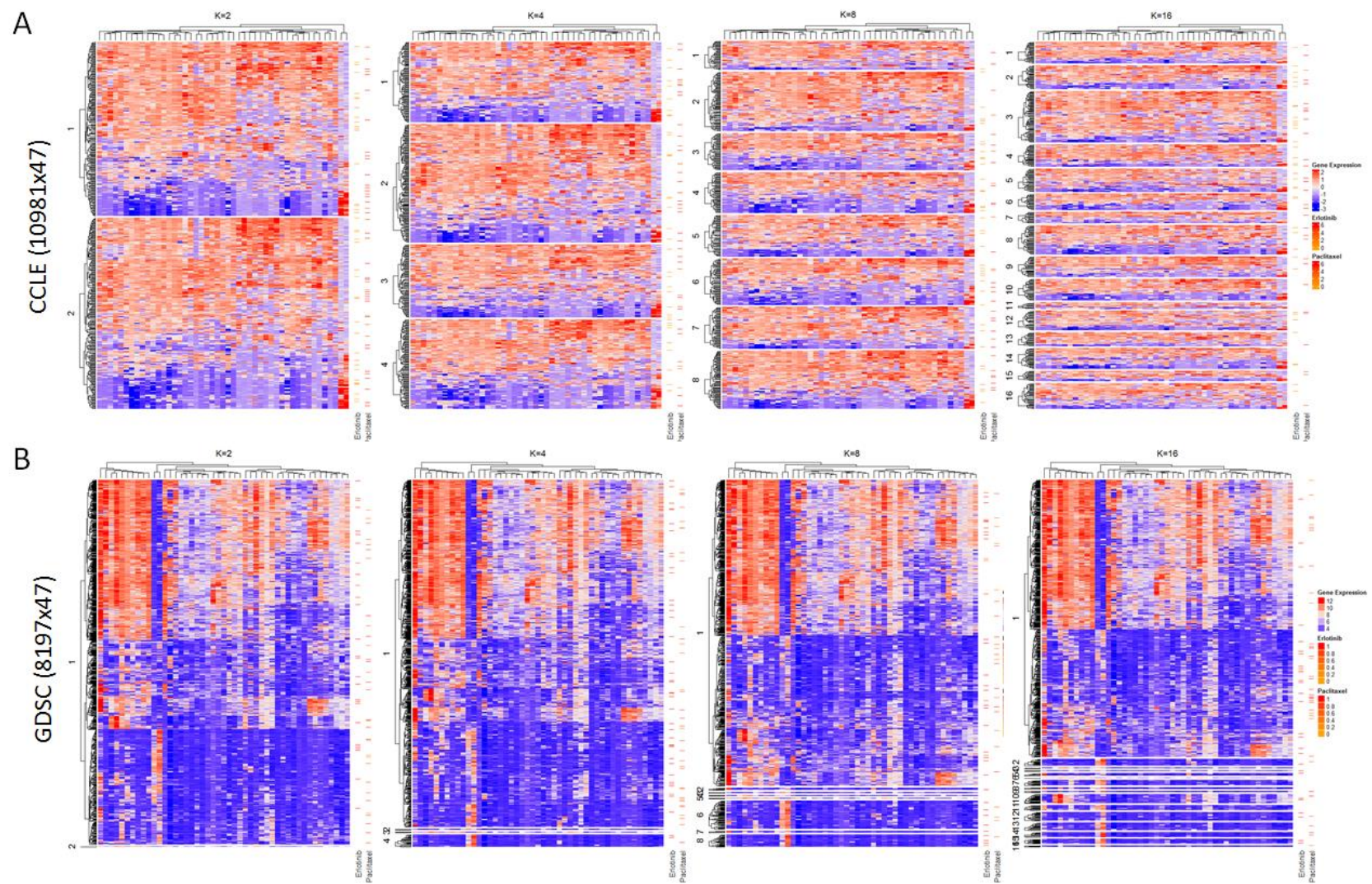

Figure S2. Alternative visualizations and unsupervised clustering of CCLE and GDSC cell lines on the basis of their 47-gene profiles. Related to Figures 2D and 4A. Spectral clustering analysis was applied using the SNFtool (Wang et al., 2014) to independently explore the potential of the 47 genes' expression data to segregate (cell line-drug experiment) samples. A. CCLE and B. GDSC results. In A. and B., rows and columns in each heatmap represent samples and genes respectively, and color represents gene expression intensity. To facilitate visualization, clustering results for different numbers of clusters (K) are provided as independent plots. Note that the order of the rows in each clustering (plot) is not preserved. In each plot, additional columns (right side) representing the drug sensitivity of the samples against Erlotinib ad Paclitaxel are illustrated. 
bioRxiv preprint doi: https://doi.org/10.1101/237727; this version posted December 21, 2017. The copyright holder for this preprint (which was not certified by peer review) is the author/funder, who has granted bioRxiv a license to display the preprint in perpetuity. It is made available under aCC-BY-NC-ND 4.0 International license.
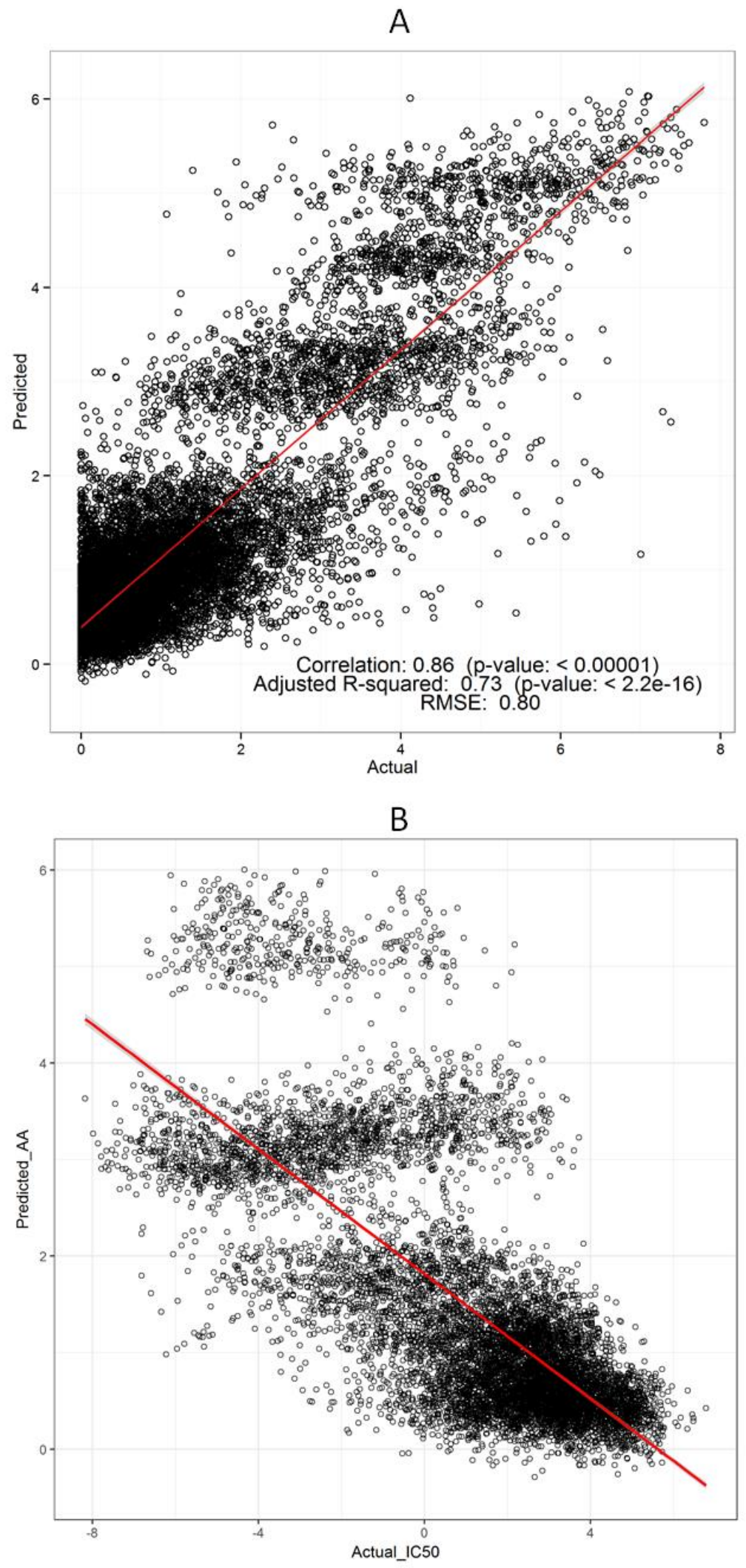

Figure S3. Predicted vs. actual sensitivity values in the CCLE and GDSC datasets Related to Figures $3 \mathrm{~A}$ and 4B. Alternative visualization to those shown in Figure 3A. A. CCLE plot ( $n=10981)$. B. GDSC plot $(n=9984)$. 

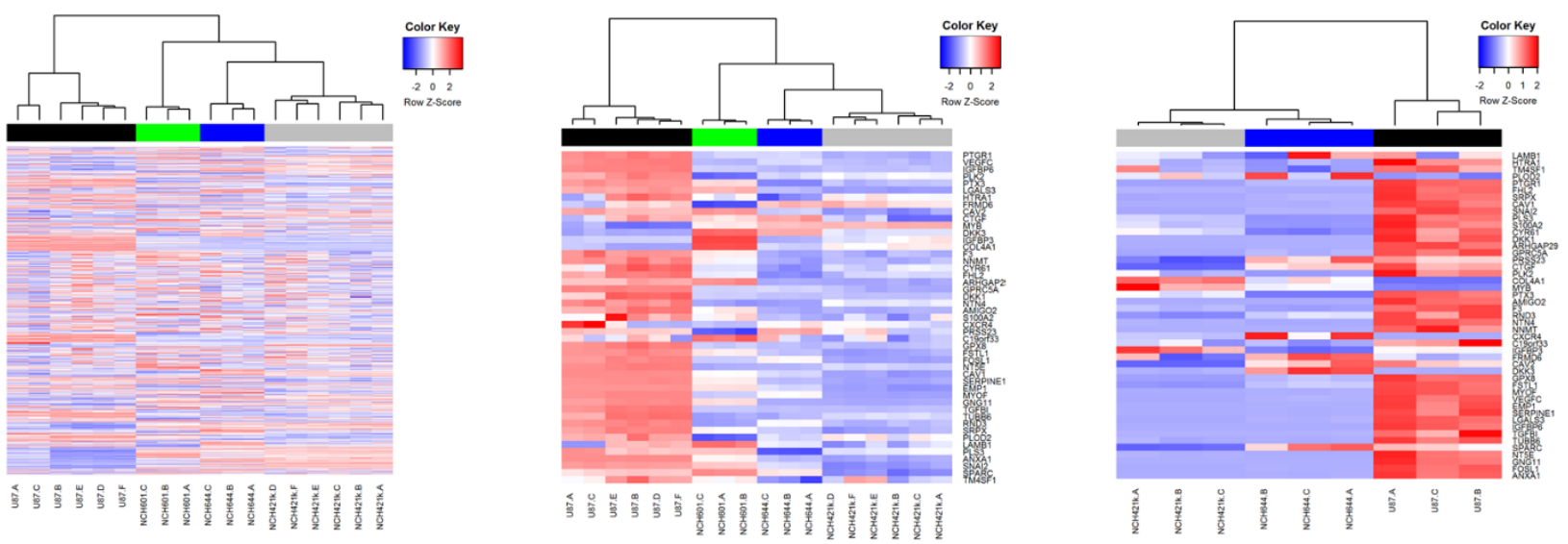

Figure S4. The 47-gene signature distinguishes cell types and is reproducible. Related to section: "Independent in vitro validation on several cell lines and compounds". Gene expression of 47 genes in 3 GBM cell lines using microarrays and qPCR. Analysis peformed to verify the robustness and platform-independent replicability of the 47-gene expression data and its capacitiy to distinguish between cell lines. 


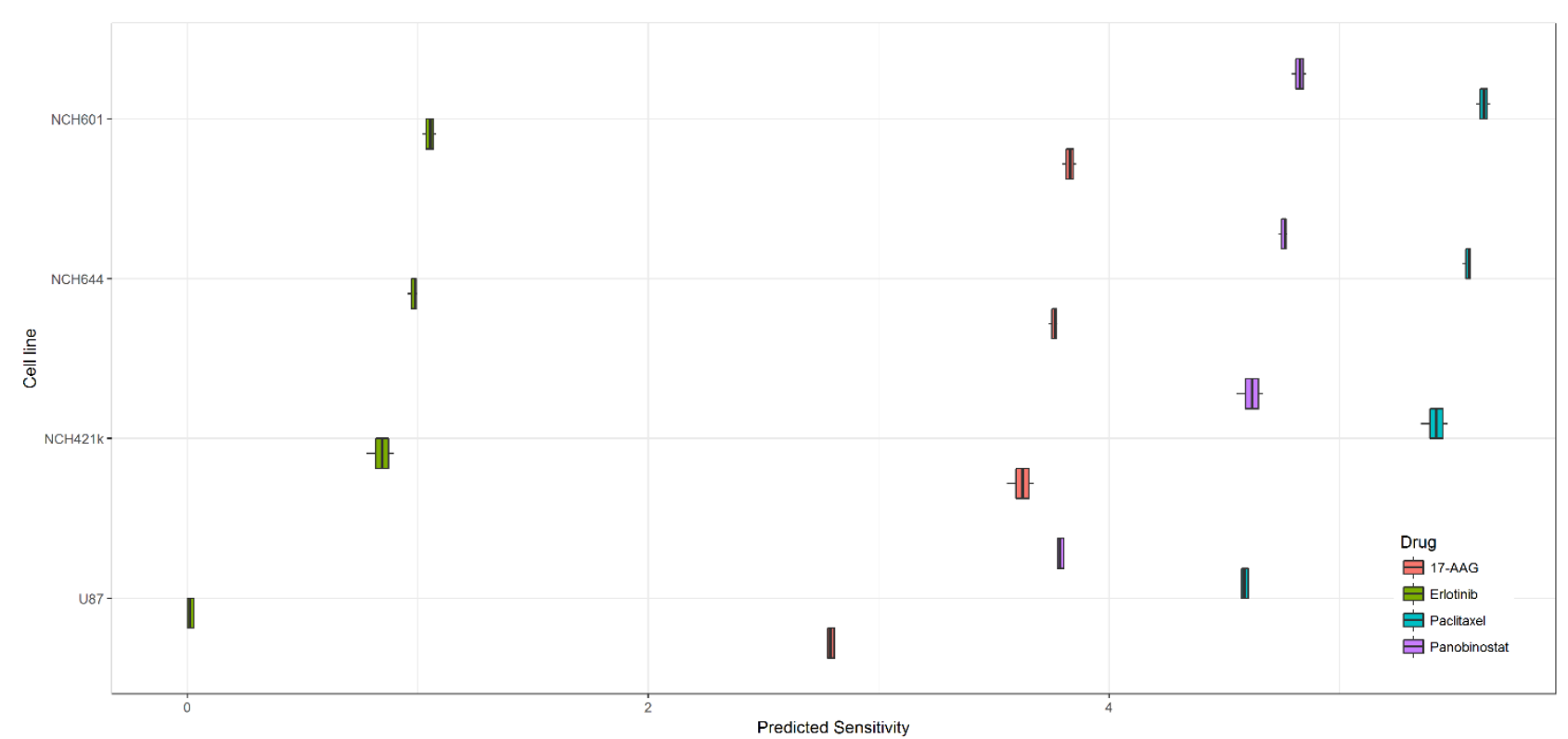

Figure S5. Boxplot summary of prediction results for 4 drugs (Erlotinib, 17-AAG, Panobinostat and Paclitaxel) and 4 GBM cell lines. Related to Figure 5B. Ech cell line type comprises multiple biological replicates (18 samples in total): $6 \mathrm{U} 87,3 \mathrm{NCH} 644,3$ $\mathrm{NCH} 601$ and $6 \mathrm{NCH} 421 \mathrm{k}$ samples.
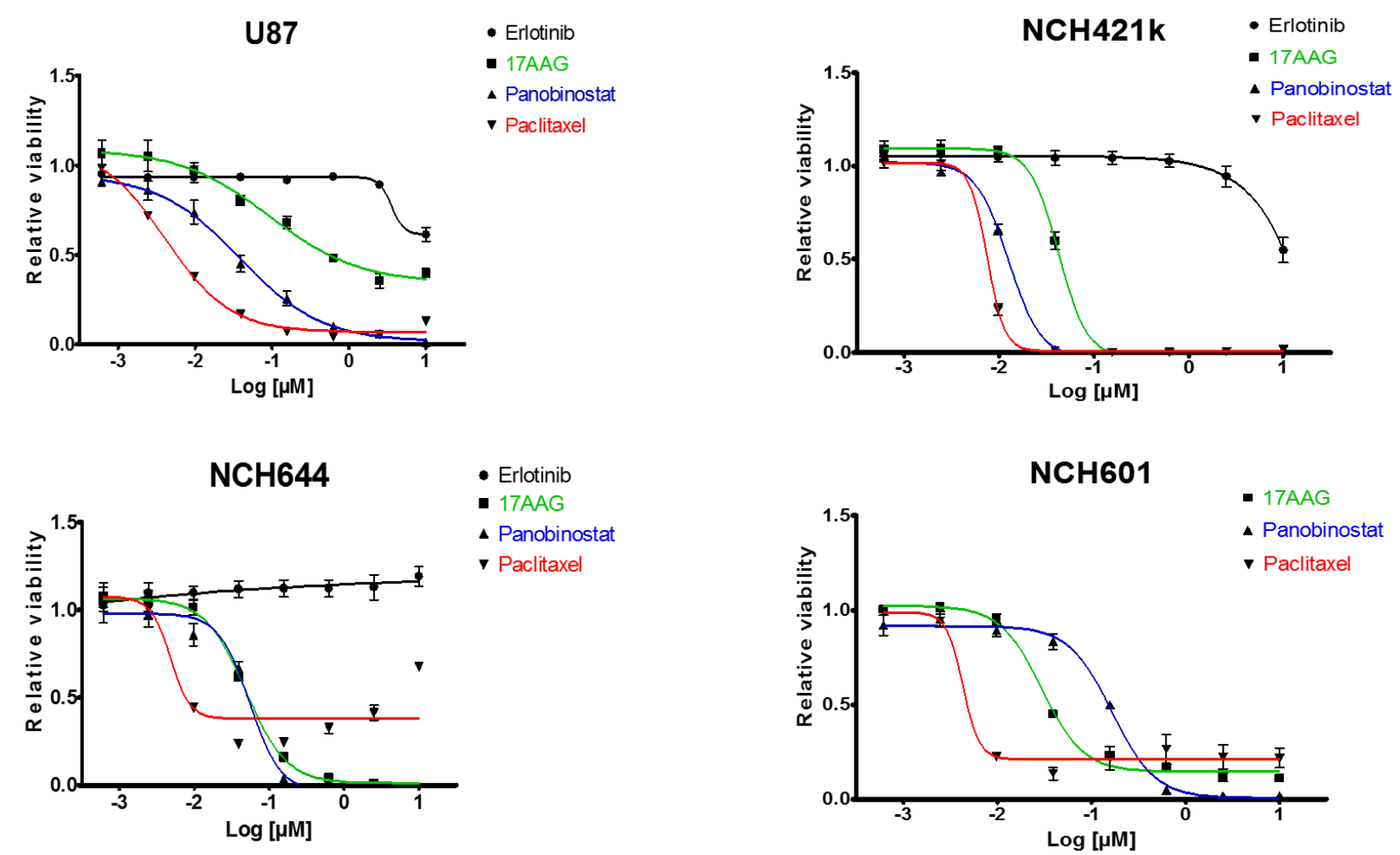

Figure S6. Drug response curves for the 4 drugs tested on the 4 GBM cell lines. Related to Figure 5C. Drugs were tested on each cell line in triplicates, and relative viability (vs. vehicle-treated samples) was measured for 8 drug concentration values (shown here as $\log [\mu \mathrm{M}])$. 
bioRxiv preprint doi: https://doi.org/10.1101/237727; this version posted December 21, 2017. The copyright holder for this preprint (which was not certified by peer review) is the author/funder, who has granted bioRxiv a license to display the preprint in perpetuity. It is made available under aCC-BY-NC-ND 4.0 International license.

\begin{tabular}{|c|c|c|}
\hline ITEM TO СНЕСК & IMPORTANCE & СНЕСКLIST \\
\hline EXPERIMENTAL DESIGN & 然 & \\
\hline Number within each group & $\mathrm{E}$ & 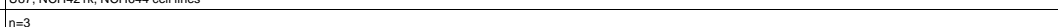 \\
\hline Assay carried out by core lab or investigator's lab? & $\mathrm{D}$ & \\
\hline $\begin{array}{l}\text { Achnowledgement of author's contributi } \\
\text { SAMPLE }\end{array}$ & D & \\
\hline Volume/mass of sample processed & 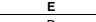 & 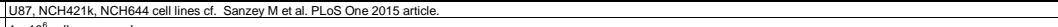 \\
\hline 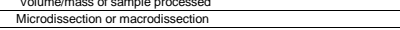 & $\frac{D}{E}$ & $1 \times 10^{\circ}$ cells per sample \\
\hline $\begin{array}{l}\text { Processing procedure } \\
\text { Iftrozen-how and how quickly? }\end{array}$ & E & Cells were washed and counted in PBS (without $\mathrm{Ca}++$ and $\mathrm{Mg}++$ ). $1 \times 106$ cells were resuspended in TRI Reagent(B), snap-frozen and then stored at \\
\hline 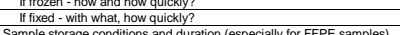 & $\frac{\mathrm{E}}{\mathrm{E}}$ & Samples were snap-riozen in TRI Reagent@ and stored at $-80^{\circ} \mathrm{C}$ \\
\hline $\begin{array}{l}\text { Sample storage conditions and duration (especially for FFPE samples) } \\
\text { NuCLEIC ACID EXTRACTION }\end{array}$ & $\mathrm{E}$ & Samples were stored in TRI Reagentit at $-80^{\circ} \mathrm{C}$ until RNA extraction \\
\hline Procedure and/or instrumentation & E & 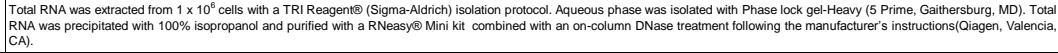 \\
\hline \begin{tabular}{|l|l|l} 
Name of kit and details of any modifications \\
SSurce ef additional reagents used \\
\end{tabular} & E E & TRI Reagent(囚 - RNeasy@ Mini kit combined with an on-column DNase treatment following the manufacturer's instructions. \\
\hline \begin{tabular}{|l|l} 
Source of addititnal reagents used \\
Details of DNase or RNAse treatment
\end{tabular} & $\frac{D}{E}$ & 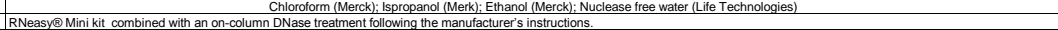 \\
\hline Contamination assessment (DNA or RNA) & E & DNase treatement + Bioanalyzer + primers flanking intron + Negative controle (RT \& qPCR) \\
\hline $\begin{array}{l}\text { Nucleic acid quantification } \\
\text { Instrument and method } \\
\end{array}$ & $\frac{E}{E}$ & Nanodrop \\
\hline \begin{tabular}{|l|l} 
Instrument and method \\
Purity (A260/A280)
\end{tabular} & $\frac{E}{D}$ & $\frac{\text { Nanodrop }}{\text { A sample: Prutit(A26/A280) } \approx 2}$ \\
\hline 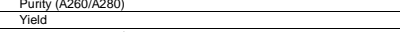 & D & A sample: Purity (A2600/A280) $\approx 2$ \\
\hline $\begin{array}{l}\text { RNAintegnily methodinstrument } \\
\text { RiN/RQl or Cq of } 3^{\prime} \text { and } 5^{\prime} \text { 'transcripts }\end{array}$ & $\frac{\mathrm{E}}{\mathrm{E}}$ & $\begin{array}{l}\text { Bioanalyzer } \\
\text { RNA sample: RIN } \geq 9\end{array}$ \\
\hline Electrophoresesis traces & L & N/A \\
\hline 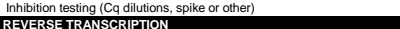 & $\mathrm{E}$ & The standard curve perfomed to check primers efficiency has been considerred sufficient to rule out the presence of finhibitiors of reverse transcrition activity or PCR. \\
\hline Complete reaction conditions & $\mathrm{E}$ & 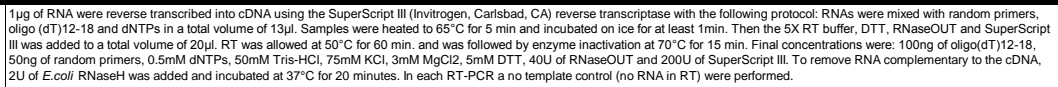 \\
\hline 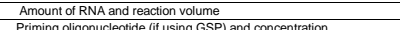 & E & $1 \mu \mathrm{g} \mathrm{RNA} / 20 \mu \mathrm{\mu}$ reaction volume \\
\hline \begin{tabular}{|l} 
Priming oligonucleotide (if Iusing GSPP and concentration \\
Reverse transcriptase end concentration
\end{tabular} & $\frac{E}{E}$ & 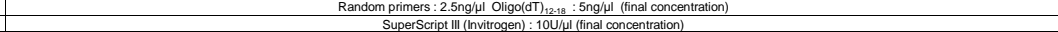 \\
\hline Temperature and time & E & 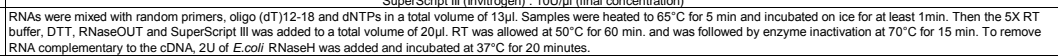 \\
\hline Manutacturer of reagents and catalogue numbers & D & $\begin{array}{l}\text { Life Technologies: SuperScript III (Cat.18080-085), Oligo(dT) 12118 primer (18418-012), Random primers (Cat. 48190-011), 10mM dNTP Mix (18427-013), RNaseOUT 40U/1/4l (10777-019), } \\
\text { E.coli RNaseH (AM2293) }\end{array}$ \\
\hline Cqs with and without $R T$ & $\mathrm{D}^{*}$ & N/A-DNase treatement + primers flanking intron + Melt Curve \\
\hline 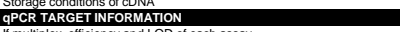 & & \\
\hline $\begin{array}{l}\text { If multiplex, efficiency and LOD of } \\
\text { Sequence accession number }\end{array}$ & $\bar{E}$ & see additional file $X-A$ \\
\hline Location of amplicon & D & 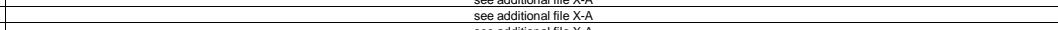 \\
\hline Amplicon length & $\frac{E}{E}$ & see additional flie $X-A$ \\
\hline 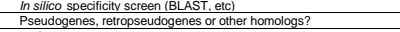 & $\frac{E}{D}$ & Beacon Designer Pro 8.10 software (Premier Biosofft) + NCBI BLAST tool \\
\hline \begin{tabular}{|l|l|} 
Sequence alignment \\
Secondary structure analysis of amplicon \\
\end{tabular} & $\frac{D}{D}$ & \\
\hline \begin{tabular}{|l|l} 
Location of each primer by vexon or intron (if applicable) \\
\end{tabular} & L & see additional flie $X-A$ \\
\hline $\begin{array}{l}\text { What splice variants are targeted? } \\
\text { qPCR OLIGONUCLETIDES }\end{array}$ & & see additional flie X-A ct. \\
\hline Primer sequences & $\mathrm{D}$ & $\frac{\text { see additional file } X-A}{N / A}$ \\
\hline $\begin{array}{ll}\text { Probe sequences } \\
\text { Location and identity of any modifications }\end{array}$ & D** & $\mathrm{N} / \mathrm{A}$ \\
\hline $\begin{array}{l}\text { Location and identity of any modifications } \\
\text { Manutacturer of oligonucleotides }\end{array}$ & $\frac{E}{D}$ & EUROGENTEC (Seraing, Belgium) \\
\hline $\begin{array}{l}\text { Purification method } \\
\text { qPCR POTOCOL }\end{array}$ & & RP-Cartridge - Gold \\
\hline Complete reaction conditions & E & 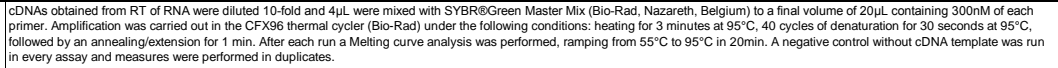 \\
\hline \begin{tabular}{|l|l|} 
Reaction volume and amount of cDNADNA \\
Primer, (probe). Mg++ and dNTP concentrations \\
\end{tabular} & E & $4 \mu \mathrm{l}$ cDNa diluted 10 fold $/ 20 \mu$ reaction volume \\
\hline \begin{tabular}{|l} 
Primer, (Probeb), Mt+ +and dNTP Poncentrations \\
Polymerase ididntity and concentration
\end{tabular} & $\mathrm{E}$ & 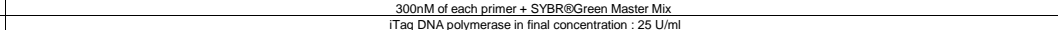 \\
\hline 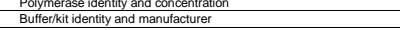 & E & 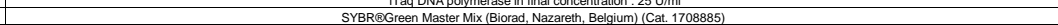 \\
\hline \begin{tabular}{|l} 
Exact chemical constitution of the buffer \\
AAditives SYYB Green DMSO pet \\
\end{tabular} & $\frac{D}{D E}$ & 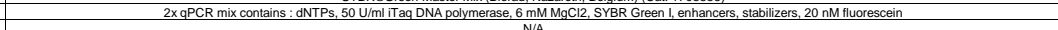 \\
\hline 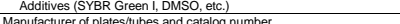 & E & \\
\hline Manulacturer of platess tubes and catalog number & & BioRad-HSP9655 \\
\hline Complete thermocycling parameters & $\mathrm{E}$ & 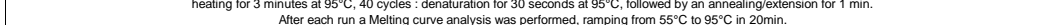 \\
\hline 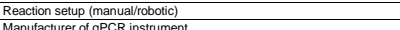 & D & \\
\hline $\begin{array}{l}\text { Manutacturer of qPCR inst } \\
\text { qPCR VALIDATION }\end{array}$ & & \\
\hline Specificity (gel, sequence, melt, or digest) & $\mathrm{E}$ & 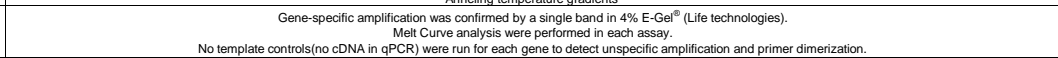 \\
\hline For SYBR Green I, Cq of the NTC & E & No amplifiction signal detected \\
\hline $\begin{array}{l}\text { Standard curves sith hlope and } y \text {-intercept } \\
\text { PCR efficiency calculated rom slope }\end{array}$ & $\frac{E}{E}$ & 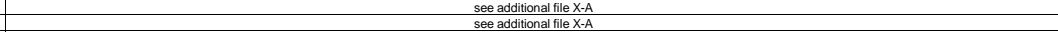 \\
\hline Confidence interval for PCR efficiency or standard error & & \\
\hline 12 of standard curve & $\frac{E}{E}$ & see additional flie $X-A$ \\
\hline $\begin{array}{l}\text { Linear dyyanim range limit } \\
\text { Cq variation at lower limit } \\
\end{array}$ & $\frac{E}{E}$ & 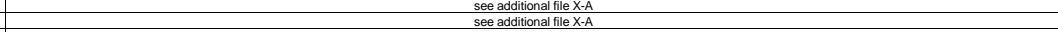 \\
\hline \begin{tabular}{|l} 
Confididence intevals throughout range \\
Evidence for limit of detection
\end{tabular} & $\mathrm{E}$ & see additional file X-A \\
\hline & $\mathrm{E}$ & N N \\
\hline $\begin{array}{l}\text { DATA ANALLSIS } \\
\text { PCR analysis rogram (source version) }\end{array}$ & & \\
\hline Cq method determination & $\mathrm{E}$ & 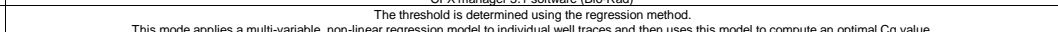 \\
\hline \begin{tabular}{|l|l|l} 
Outlier identification and disposition \\
\end{tabular} & E & Bad replicates were retested and measurementst below LOD were discard \\
\hline $\begin{array}{l}\text { Ressults of NTCS } \\
\text { Justification of number and choice of reference genes }\end{array}$ & & 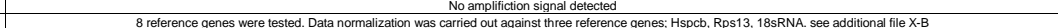 \\
\hline Description of normalisation method & E & Normalized expression was calculated using the CFX manager 3.1 software (Biorad) via the $\Delta \Delta-C q$ method, \\
\hline Number and concordance of biological replicates & D & \\
\hline Number and stage (RT or GPCR) of fechnical replicates & E & GPCR reactions were pertormed in duplicates \\
\hline Repeatability (intra-assay variation) & $\mathrm{E}$ & 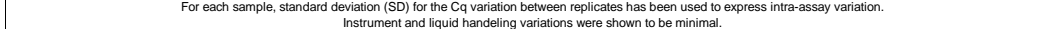 \\
\hline Reproducibility (inter-assay variation, \%CV) & D & \\
\hline Power analysis & D & \\
\hline $\begin{array}{l}\text { Staistical methods tor result significance } \\
\text { Sottware (source version) }\end{array}$ & $\mathrm{E}$ & $\begin{array}{l}\text { Refer to Methods section of aricle } \\
\text { Refer to Methods section of aricle }\end{array}$ \\
\hline Cq or raw data submission using RDML & & \\
\hline
\end{tabular}

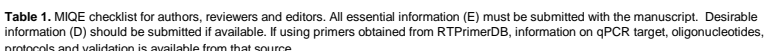

protocols and validation is avaliablet rom that source

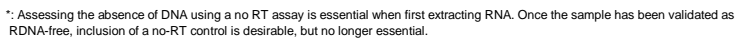

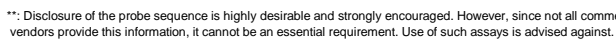


A- MIQE $q P C R$ primers informations

\begin{tabular}{|c|c|c|c|c|c|c|c|c|}
\hline Gene Name & Accession number & Foward primer sequence $\left(5^{\prime}>3^{\prime}\right)$ & Reverse primer sequence $\left(5^{\prime}>3^{\prime}\right)$ & Primers location & Amplicon (pb) & Standard curves & PCR efficiency $(\%)$ & $\mathrm{r} 2$ \\
\hline 18SRNA & NR_003286 & CAGGATTGACAGATTGAT & TTATCGGAATTAACCAGAC & only one exon & & $y=2.977 \times-3.175$ & $106.50 \%$ & 0.999 \\
\hline AMIGO2 & NM_001143668; NM_181847 & TTCTGGATTCTGAGTGGATTC & TGCTGGTGATGTTGTTATGA & F :E2(ou3) - R: E2(ou3) (same exon) & 78 & $y=19.694 \times-3.325$ & $99.90 \%$ & 0.996 \\
\hline ANXA1 & NM_000700 & TCGCAGAGTGTTTCAGAA & TCTCAATGTCACCTTTCAAC & F:E8/9 (Intron 887pb) - R:E9 & 86 & $y=11.534 x-3.189$ & $105.80 \%$ & 0.999 \\
\hline ARHGAP29 & $\begin{array}{ll}\text { NM_ } 004815 \\
1020350\end{array}$ & AAGAACACTGACTCTATCG & CTCCAATTCCAAGTTAAGC & \begin{tabular}{|l|l|} 
F:E7 - R:E7/8 (Intron 1066pb) \\
5 C.
\end{tabular} & 108 & $y=18.879 \times-3.448$ & $95.00 \%$ & 0.998 \\
\hline C19ort33 & NM_033520 & TCCAAAGCAAGGACACCA & TGGGACTTCACATCCGTG & F:E 2/3 (Intron 133pb) - R:E3/4 (Intron 158pb) & 75 & $y=18.655 x-3.268$ & $102.30 \%$ & 0.996 \\
\hline CAV1 & $\begin{array}{l}\text { NM_001 172895; NM_001172896; } \\
\text { NM_001172897; NM_001753 }\end{array}$ & AGATCGACCTGGTCAACC & GCAATCACATCTTCAAAGTCAATC & F:E 2(ou 1) - R:E 2/3 (ou1/2) (Intron 32256pb) & 76 & $y=14.398 x-3.308$ & $100.60 \%$ & 0.999 \\
\hline CAV2 & $\begin{array}{l}\text { NM_001206747; NM_001206748; } \\
\text { NM 001233; NM 198212 }\end{array}$ & CAAGTCTATAATGTGAGTAGT & TTATTCCAGTTCAATCATCA & F : E3 - R: E3 (3'UTR) & 190 & $y=18.621 x-3.566$ & $90.70 \%$ & 0.997 \\
\hline COL4A1 & NM_001845 & AGGGACAAATGGGCTTAA & TTCTTGAACTTGAGCTTGT & F:E11/12 (Intron 501pb) - R:E13 (Intron 1359pb) & 101 & $y=18.405 x-3.331$ & $99.60 \%$ & 0.998 \\
\hline CTGF & NM_ 001901 & GCTGACCTGGAAGAGAAC & AAACTTGATAGGCTTGGAGAT & F:E4 - R:E5 (Intron 388pb) & 75 & $y=17.593 x-3.42$ & $96.10 \%$ & 0.996 \\
\hline $\begin{array}{l}\text { CXCR4 } \\
\text { CYR61 }\end{array}$ & NM_001008540; NM_003467 & 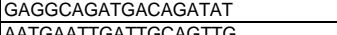 & AATACCAGGCAGGATAAG & 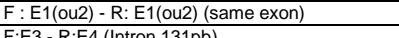 & $\frac{105}{89}$ & $\begin{array}{l}y=17.432 x-3.254 \\
y=17355 x-3.158\end{array}$ & $102.90 \%$ & 0.996 \\
\hline $\begin{array}{l}\text { CYR61 } \\
\text { DKK1 } 1\end{array}$ & \begin{tabular}{|l|l|} 
NM_001554 \\
NM_012242
\end{tabular} & $\begin{array}{l}\text { AATGAATTGATTGCAGTTG } \\
\text { TATCACACCAAAGGACAA }\end{array}$ & $\begin{array}{l}\text { TGTAAAGGGTTGTATAGGA } \\
\text { GTCTAGCACAACACAATC }\end{array}$ & 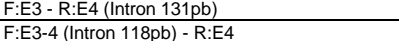 & $\frac{89}{76}$ & $\begin{array}{l}y=17.355 x \times 3.158 \\
y=19.432 \times-3.335\end{array}$ & $\frac{107.30 \%}{99.50 \%}$ & 0.9989 \\
\hline DKK3 & $\begin{array}{l}\text { NM_015881; NM_013253; } \\
\text { NM 001018057 }\end{array}$ & AAAGCATCATCAGAAGTG & TGTTGGTTATCTTGTGAAT & F:E3 - R:E3/4 (Intron 3520pb) & 124 & $y=17.042 \times-3.292$ & $101.30 \%$ & 0.999 \\
\hline EMP1 & \begin{tabular}{|l|l|l|l|l} 
NM_ 001423 \\
\end{tabular} & AATGTCTGGTTGGTTTCC & GCATCTTCACTGGCATAT & $F: E 2 / 3$ (Intron 1890pb) - R:E3/4 (Intron 105pb) & 104 & $y=13.964 x-3.231$ & $104.00 \%$ & 0.999 \\
\hline F3 & NM_001178096; NM_001993 & CGTCAATCAAGTCTACAC & TTCACATCCTTCACAATC & F:E2 - R:E3 (Intron 4092pb) & 117 & $y=19.464 x-3.087$ & $110.80 \%$ & 0.998 \\
\hline FHL2 & $\begin{array}{l}\text { NM_001039492; NM_001450; } \\
\text { NM 20155:NM 201557 }\end{array}$ & AGACTGCTATTCCAACGA & ССTTGTACTCCATCTTGC & F:E3/4/5 - R:E 4/5/6 (Intron 5819pb) & 86 & $y=15.448 \times-3.295$ & $101.20 \%$ & 0.999 \\
\hline FOSL1 & NM_ 005438 & TCCCTAACTCCTTTCACC & CTGCTACTCTTGCGATGA & $F: E 4-R: E 4$ & 86 & $y=13.047 x-3.245$ & $103.30 \%$ & 0.999 \\
\hline FRMD6 & $\begin{array}{l}\text { NM_001042481; NM_001267046; } \\
\text { NM 001267047:NM 152330 }\end{array}$ & CTACATCACAGAGGACAT & GACCCAATTTCTTTCACA & $F: E 3 / 12 / 13$ - R:E 4/13/14 (Intron 3698pb) & 75 & $y=18.423 x-3.454$ & $94.80 \%$ & 0.999 \\
\hline \begin{tabular}{|l|} 
FSTL1 \\
GNG11
\end{tabular} & 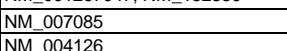 & $\begin{array}{l}\text { CTGCCATCAATATTACAAC } \\
\text { GAAGATTTGCAAGAGAAG } \\
\end{array}$ & $\begin{array}{l}\text { TTCATCAGACAGTTCAAT } \\
\text { ACATTTAGACACTTGTTGT }\end{array}$ & \begin{tabular}{|l|}
$\mathrm{F}: E 7$ - R:E8 (Intron 1498pb) \\
$\mathrm{F}: \mathrm{E} 1$ - R:E1/2 (Intron 3857pb)
\end{tabular} & $\frac{92}{90}$ & $\begin{array}{l}y=15.296 \times-3.351 \\
y=12.903 \times 3 \times 3.283\end{array}$ & $\frac{98.80 \%}{101.60 \%}$ & $\begin{array}{ll}0.992 \\
1.000\end{array}$ \\
\hline GPRC5A & NM 003979 & CTCAACTCGTGAAGAAGA & GAAAATGTGTGGAATAGGG & F:E2-R:E3 (Intron 2912pb) & $\frac{50}{117}$ & $\begin{array}{l}y=18.905 x \times-3.283 \\
y=18.350 x-3.274\end{array}$ & $102.00 \%$ & 0.0909 \\
\hline GPX8 & NM 001008397 & CTTCCACAAGATTAAGATTC & GTTGACAAGATACTTCCA & F:E2 - R:E3 (Intron 2799pb) & 109 & $y=15.841 x-3.334$ & $99.50 \%$ & 0.999 \\
\hline HSPCB & $\begin{array}{l}\text { NM_001271969; NM_001271970; } \\
\text { NM_001271971; NM_001271972; } \\
\text { NM_007355 }\end{array}$ & AAGCATTCTCAGTTCATA & ТСТTСТTСТTATССTTACC & F:E5 - R:E 6 (Intron 136) & 193 & $y=11.415 x-3.323$ & $99.90 \%$ & 0.999 \\
\hline \begin{tabular}{|l|} 
HTRA1 \\
IGFBP3
\end{tabular} & NM_ 002775 & $\begin{array}{l}\text { ATCATCAACTATGGAAAC } \\
\text { CGGGAGACAGATATGGT }\end{array}$ & $\begin{array}{l}\text { GATACCAATATACTTCTTCT } \\
\text { CAGCACATTGAGGAACTT }\end{array}$ & 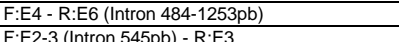 & 192 & $y=15.354 x-3.368$ & $\begin{array}{l}98.10 \% \\
0.70 \%\end{array}$ & 1.000 \\
\hline $\begin{array}{l}\text { IIFBP3 } \\
\text { IGFBP6 }\end{array}$ & $\mid \begin{array}{l}\text { NM_000598; NM_001013398 } \\
\text { NM 002178 }\end{array}$ & $\begin{array}{l}\text { CCCTCCCAGCCCAATTCT } \\
\end{array}$ & $\begin{array}{l}\text { lAGCACAIGAGGAAACII } \\
\text { CAGCACTGAGTCAGATGTCT }\end{array}$ & 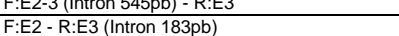 & $\frac{75}{75}$ & $\begin{array}{l}y=19.317 x-3.354 \\
y=14.322 x-3.307\end{array}$ & $\frac{98.70 \%}{10060 \%}$ & 0.999 \\
\hline LAMB1 & NM 002291 & ATTATCTGACACAACTTC & AATACTTGGTAATGCTATC & F:E26 - R:E27 (Intron 1436pb) & $\frac{15}{164}$ & $\begin{array}{l}y=14.02 x-0.007 \\
y=15.632 x-3.315\end{array}$ & $100.30 \%$ & 0.999 \\
\hline LGALS3 & NM_001177388; NM_002306 & ATGCTGATAACAATTCTGG & CAAACAATGACTCTCCTG & F:E4 - R:E5 (Intron 2220pb) & 131 & $y=14.376 x-3.436$ & $95.50 \%$ & 0.999 \\
\hline MYB & $\begin{array}{l}\text { NM_001 130172; NM_001130173; } \\
\text { NM_0116165; NM_00161655; } \\
\text { NM_001161658; NM_001161659; }\end{array}$ & CAACGACTATTCCTATTACC & CTGAGGGACATTGACTAT & F:E6 - R:E7 (Intron 1279pb) & 100 & $y=16.785 \times-3.308$ & $100.60 \%$ & 0.998 \\
\hline MYOF & NM_013451; NM_133337 & ATAGAAGACACGAGATACAC & GCTITCGGATCTGAGTAT & F:E21/22 (Intron 2367pb) - R:E 22 & 79 & $y=15.903 x-3.228$ & $104.10 \%$ & 1.000 \\
\hline $\begin{array}{l}\text { NNMT } \\
\text { NTMET }\end{array}$ & \begin{tabular}{|l|l|} 
NM_006169 \\
\end{tabular} & CAGTGGTGACCTAT & CCTGTC & F:E4 - R:E5 (Intron 138860b) & 75 & $y=17.363 x-3.312$ & $100.40 \%$ & 1.000 \\
\hline NT5E & NM_001204813; NM_002526; & $\begin{array}{l}\text { GGAATCGITGGATACACT } \\
\end{array}$ & ACTTATCTACTTCAGGTTGT & $\begin{array}{l}\text { F:E2 - R:E3 (Intron 3954pb) } \\
\text { FEFT }\end{array}$ & $\frac{106}{121}$ & $y=13.536 x-3.344$ & $95.10 \%$ & 1.000 \\
\hline $\begin{array}{l}\text { NTN4 } \\
\text { PLK2 }\end{array}$ & NM_021229 & $\begin{array}{l}\text { |CTGGAAGATGATGTIGTC } \\
\text { GGATGTATCGGATATT }\end{array}$ & GGTTCTCTGTATCGTATG & F:E2 - R:E3 (Intron 48794pb) & $\frac{121}{78}$ & $y=20.842 x-3.313$ & $100.40 \%$ & 0.994 \\
\hline $\begin{array}{l}\text { PLK2 } \\
\text { PLOD2 }\end{array}$ & 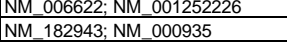 & $\begin{array}{l}\text { GGATGCTATTCGGATGAT } \\
\text { CTAGCAGACAGGTATCCT }\end{array}$ & \begin{tabular}{|l} 
ATGGTACTGTCTTCAAGG \\
GAACTATACGGTTGACATAT
\end{tabular} & $\begin{array}{l}F: E 10 \text { - R:RE11 (Intron 2460b) } \\
F: E 4: E \text { (Intron 36400b) }\end{array}$ & $\frac{78}{94}$ & $\begin{array}{l}y=17.666 x \times 3.423 \\
y=16.960 \times-3.311\end{array}$ & $\frac{95.90 \%}{100.40 \%}$ & $\begin{array}{l}0.997 \\
0.999\end{array}$ \\
\hline PLS3 & $\begin{array}{l}\text { NM_001 136025; NM_001172335; } \\
\text { NM_001282337; NM_001282338; } \\
\text { NM_005032 }\end{array}$ & GCTGATGAGAAGATATACC & CTCTGAATGGAAGTTGAT & F:E13/14 (Intron 1014pb) - R:E14 & 129 & $y=15.001 \times-3.349$ & $98.90 \%$ & 0.999 \\
\hline PRSS23 & NM_007173 & CTCGGCGCGGAACAG & CCAACAGCACAGAGCAGAA & F:E1/2 (Intron 6979pb) - R:E2 & 79 & $y=18.777 x-3.346$ & $99.00 \%$ & $\begin{array}{ll}0.997 \\
\end{array}$ \\
\hline PTGR1 & $\begin{array}{l}\text { NM_001146108; } \\
\text { NM 001161610:NM } 012212\end{array}$ & GTTGGCTATCCTACTAAT & CATCATTGTATCACCTTC & F:E2 - R:E4 (Intron 3049/1239pb) & 153 & $y=15.472 \times-3.356$ & $98.60 \%$ & 0.999 \\
\hline PTX3 & NM_002852 & TGAATTाGGACAACGAAATAGAC & ETGCTCCTGAC & F:E1 - R:E2 (Intron 449pb) & 84 & $y=20.26 x-3.573$ & $90.50 \%$ & 0.997 \\
\hline RND3 & NM_001254738; NM_005168 & TGTTAGTACATTAGTAGAG & AGCATTCGATATAAGTAG & F:E5 - R:E6 (Intron 1388pb) & 107 & $y=18.532 \times-3.148$ & $107.80 \%$ & 0.996 \\
\hline RPS13 & NM_001017 & GTCCCCACTTGGTTGAAG & CCATGTGAATCTCTCAGGAT & $\mathrm{F}: E 2-3$ (Intron182) - R:E3 & 113 & $y=12.185 x-3.344$ & $99.10 \%$ & 1.000 \\
\hline S100A2 & NM_005978 & CAAGTTCAAGCTGAGTAAG & CTCCTCATCCACTTTCTC & F:E2 - R:E3 (Intron 2142pb) & 85 & $y=18.328 x-3.244$ & $103.40 \%$ & 0.999 \\
\hline SERPINE1 & NM_000602 & TGGGAATGAC & GAGGCT & F:E6 - R:E 6/7 (Intron 120pb) & 75 & $y=14.046 \times-3.274$ & $102.00 \%$ & 1.000 \\
\hline SNAI2 & NM_003068 & ACACATACAGTGATTATTTCC & GTAGTCCACACAGTGATG & $\mathrm{F}: \mathrm{E} 1-2 / 2-3$ (Intron 745pb) - R:E2/3 & 113 & $y=15.391 x-3.262$ & $102.60 \%$ & 1.000 \\
\hline SPARC & NM_003118 & AGGTGACTGAGGTATCTGT & TGGTTCTGGCAGGGATTT & $\mathrm{F}: \mathrm{E}$ 3/4 (Intron 1395pb) - R:E4/5 (Intron 1434pb) & 115 & $y=13.713 x-3.308$ & $100.60 \%$ & 0.996 \\
\hline SRPX & $\begin{array}{l}\text { NM_0011770750; NM__01170751; } \\
\text { NM 001170752; NM 006307 }\end{array}$ & ATTCTTACTGATGTCATTCT & TCTGTCATAGACTGTGTA & F:E 4/5 (Intron 3714pb) - R:E 5 & 90 & $y=14.738 \times-3.142$ & $108.10 \%$ & 0.999 \\
\hline TGFBI & NM 000358 & $\begin{array}{l}\text { AGAAGGTTATTGGCACTAAT } \\
\end{array}$ & GCTGATGACTGTTGATTTG & F:E2 - R:E 2/3 (Intron 10196pb) & 89 & $y=14.290 x-3.345$ & $99.00 \%$ & 0.999 \\
\hline TM4SF1 & NM_014220 & ATTGTGGAATGGAATGTATC & ATATTC & F:E4 - R:E4/5 (Intron 1806pb) & 138 & $y=15.462 x-3.278$ & $101.90 \%$ & 0.999 \\
\hline TUBB6 & NM_032525 & AGAGAATCAACGTCTACTACAATG & GGCTCTAAGTCCACCAGG & $\begin{array}{l}\text { F:E2 - R:E3 (Intron 2147pb) } \\
\text { :CE2 R: }\end{array}$ & 76 & $y=14.063 x-3.346$ & $99.00 \%$ & 1.000 \\
\hline VEGFC & NM_005429 & AAGGACAGAAGAGACTAT & CACATCTATACACACCTC & F:E2 - R:E3 (Intron 1564pb) & 118 & $y=16.637 x-3.374$ & $97.90 \%$ & 0.999 \\
\hline
\end{tabular}

B- MIQE data analysis informations 


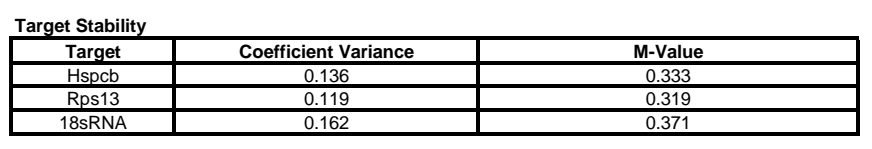

Average Coefficient Variance: : 0.139

Average M-Value: : 0.341

Coefficient of Variation (CV) of normalized reference gene relative quantities. A lower CV value denotes higher stability 


\begin{tabular}{|c|c|}
\hline $\begin{array}{l}\text { Linear dynamic range } \\
\end{array}$ & Cq variation at lower limit \\
\hline from $1.00 \mathrm{E}-01$ cDNA dilution to $1.00 \mathrm{E}-08$ & $28.24 \pm 0.389$ \\
\hline from 1.00E-01 cDNA dilution to $1.56 \mathrm{E}-03$ & $28.97 \pm 0.242$ \\
\hline from 1.00E-01 cDNA dilution to $2.96 \mathrm{E}-05$ & $25.89 \pm 0.061$ \\
\hline 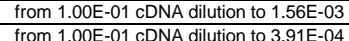 & $\frac{28.52 \pm 0.097}{2964+0.258}$ \\
\hline from 1.00E-01 CDNA dilution to $1.00 \mathrm{E}-05$ & $30.91 \pm 0.323$ \\
\hline from $1.00 \mathrm{E}-01 \mathrm{cDN}$ d dilution to $3.91 \mathrm{E}-04$ & $30.57 \pm 0.095$ \\
\hline from $1.00 \mathrm{E}-01 \mathrm{CDNA}$ dilution to $1.56 \mathrm{E}-03$ & $27.67 \pm 0.007$ \\
\hline from 1.00E-01 cDNA dilutition to 3.91E-04 & $29.05 \pm 0.198$ \\
\hline from 1.00E-01 CDNA dilution to $3.91 \mathrm{E}-04$ & $28.35 \pm 0.255$ \\
\hline from 1.00E-01 cDNA dilution to 7.72E-05 & $30.17 \pm 0.209$ \\
\hline from 1.00E-01 cDNA dilution to $1.56 \mathrm{E}-03$ & $28.75 \pm 0.093$ \\
\hline from $1.00 \mathrm{E}-01 \mathrm{cDNA}$ dilution to $1.60 \mathrm{E}-04$ & $29.61 \pm 0.057$ \\
\hline from $1.00 \mathrm{E}-01 \mathrm{CDNA}$ dilution to $1.00 \mathrm{E}-04$ & $26.82 \pm 0.109$ \\
\hline from 1.00E-01 cDNA dilution to $3.91 \mathrm{E}-04$ & $29.89 \pm 0.118$ \\
\hline from $1.00 \mathrm{E}-01 \mathrm{CDNA}$ dilution to $7.72 \mathrm{E}-05$ & $29.07 \pm 0.121$ \\
\hline from 1.00E-01 cDNA dilution to $1.00 \mathrm{E}-05$ & $29.27 \pm 0.35$ \\
\hline from $1.00 \mathrm{E}-01 \mathrm{cDNA}$ dilution to $3.91 \mathrm{E}-04$ & $30.2 \pm 0.140$ \\
\hline from $1.00 \mathrm{E}-01 \mathrm{CDNA}$ dilution to $1.00 \mathrm{E}-05$ & $32.48 \pm 0.861$ \\
\hline from 1.00E-01 cDNA dilution to $1.00 \mathrm{E}-05$ & $29.32 \pm 0.249$ \\
\hline from $1.00 \mathrm{0}-01$ cDNA dilution to $8.00 \mathrm{E}-04$ & $28.51 \pm 0.063$ \\
\hline from 1.00E-01 cDNA dilution to $1.95 \mathrm{E}-04$ & $28.18 \pm 0.26$ \\
\hline from $1.00 \mathrm{E}-01 \mathrm{CDNA}$ dilution to $1.98 \mathrm{E}-06$ & $30.55 \pm 0.236$ \\
\hline from 1.00E-01 cDNA dilution to 1.95E-04 & $27.81 \pm 0.046$ \\
\hline from 1.00E-01 CDNA dilution to 3.91E-04 & $30.69 \pm 0.038$ \\
\hline from $1.00 \mathrm{E}-01 \mathrm{CDNA}$ dilution to $1.00 \mathrm{E}-04$ & $27.54 \pm 0.008$ \\
\hline from 1.00E-01 cDNA dilution to 1.95E-04 & $27.89 \pm 0.209$ \\
\hline from 1.00E-01 cDNA dilution to 7.72E-05 & $28.58 \pm 0.21$ \\
\hline from $1.00 \mathrm{E}-01 \mathrm{CDNA}$ dilution to $1.60 \mathrm{E}-04$ & $29.31 \pm 0.285$ \\
\hline from $1.00 \mathrm{E}-01 \mathrm{CDNA}$ dilution to $2.44 \mathrm{E}-05$ & $30.81 \pm 0.018$ \\
\hline from $1.00 \mathrm{E}-01$ cDNA dilution to $8.00 \mathrm{E}-04$ & $27.59 \pm 0.083$ \\
\hline from $1.00 \mathrm{E}-01 \mathrm{cDNA}$ dilution to $1.00 \mathrm{E}-05$ & $30.7 \pm 0.112$ \\
\hline from 1.00E-01 CDNA dilution to $3.70 \mathrm{E}-03$ & $28.81 \pm 0.324$ \\
\hline from 1.00E-01 cDNA dilution to $3.91 \mathrm{E}-04$ & $29.34 \pm 0.337$ \\
\hline from $1.00 \mathrm{E}-01$ cDNA dilution to $7.72 \mathrm{E}-05$ & $30.7 \pm 0.098$ \\
\hline from $1.00 \mathrm{E}-01 \mathrm{CDNA}$ dilution to $7.72 \mathrm{E}-05$ & $28.83 \pm 0.049$ \\
\hline from $1.00 \mathrm{E}-01 \mathrm{CDNA}$ dilution to $3.91 \mathrm{E}-04$ & $30.12 \pm 0.089$ \\
\hline from $1.00 \mathrm{E}-01 \mathrm{CDNA}$ dilution to $7.72 \mathrm{E}-05$ & $29.21 \pm 0.25$ \\
\hline from $1.00 \mathrm{E}-01$ cDNA dilution to $6.25 \mathrm{E}-03$ & $28.81 \pm 0.23$ \\
\hline from 1.00E-01 cDN & $28.43 \pm 0.042$ \\
\hline from $1.00 \mathrm{E}-01$ cDNA dilution to $1.00 \mathrm{E}-04$ & $25.61 \pm 0.076$ \\
\hline from $1.00 \mathrm{E}-01 \mathrm{CDNA}$ dilution to $1.60 \mathrm{E}-04$ & $30.51 \pm 0.112$ \\
\hline from 1.00E-01 cDNA dilution to 7.72E-05 & $27.47 \pm 0.162$ \\
\hline 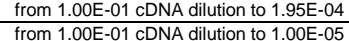 & $\frac{27.51 \pm 0.144}{30.3 \pm 0.855}$ \\
\hline from $1.00 \mathrm{E}-01 \mathrm{CDNA}$ dilution to $7.72 \mathrm{E}-05$ & $27.65 \pm 0.171$ \\
\hline from 1.00E-01 cDNA dill & \\
\hline from $1.00 \mathrm{E}-01 \mathrm{cDN}$ & \\
\hline $\begin{array}{l}\text { from } 1.00 E-01 \text { cDNA dilution to } 7.72 E-05 \\
\text { from } 1.00=-01 \text { cDNA }\end{array}$ & $\begin{array}{l}27.76 \pm 0.036 \\
27.78+0.026\end{array}$ \\
\hline
\end{tabular}

